\title{
FUNDAMENTALLÖSUNGEN UND EXISTENZ VON SCHWACHEN LÖSUNGEN LINEARER PARTIELLER DIFFERENTIALGLEICHUNGEN MIT KONSTANTEN KOEFFIZIENTEN
}

\author{
NORBERT ORTNER
}

\section{Einleitung}

1.1. In der vorliegenden Arbeit wird zuerst eine Definition einer Fundamentallösung eines linearen partiellen Differentialoperators mit konstanten Koeffizienten als Distribution ${ }^{1}$ ) gegeben. Anschließend wird von einigen konkret gegebenen Distributionen gezeigt, daß sie Fundamentallösungen gewisser Differentialoperatoren sind.

Im zweiten Teil der Arbeit wird dann eine Fundamentallösung des Laplaceoperators durch Penalisierung und Fouriertransformation gewonnen: Die Fouriertransformation liefert eine Fundamentallösung von $\Delta_{n}-4 \pi^{2} \varepsilon^{2}$. Der Grenzübergang $\varepsilon_{\downarrow} 0$ ergibt eine Fundamentallösung von $\Delta_{n}$. Ebenso kann man Fundamentallösungen etwa des Wellenoperators oder des Wärmeoperators ermitteln.

Im dritten Teil wird eine von L. Schwartz [12] angegebene Beweisskizze des Malgrange-Ehrenpreis-Theorems über die Existenz einer Fundamentallösung eines linearen Differentialoperators mit konstanten Koeffizienten unter Benutzung von Ideen von F. Treves [14] ausgearbeitet. Dieser Beweis verwendet keine funktionentheoretischen Hilfsmittel, im Gegensatz zu dem ursprünglichen von B. Malgrange [9]. Etwas abgeändert findet man den Beweis von Malgrange noch in Hörmander [6], S. 64-66, und Rudin [11], S. 195-197.

Schließlich wird die Existenz von schwachen $L^{2}$-Lösungen linearer partieller Differentialgleichungen untersucht.

1.2. Ist $\Omega$ eine offene Menge in $\boldsymbol{R}^{n}$, so sei $\mathscr{E}(\Omega)$ der Raum der unendlich oft differenzierbaren Funktionen, versehen mit der Topologie der gleichmäßigen Konvergenz bezüglich aller Ableitungen auf allen kompakten Teilmengen von $\Omega$. Ist

1) Benutzt wird die Distributionentheorie von L. Schwartz, wie sie in vielen Lehrbüchern dargestellt ist; gegebenenfalls wird auf Sätze der Monographie [15] von F. Treves hingewiesen. 
$K(\subset \Omega)$ kompakt, so sei $\mathscr{D}_{K}(\Omega)$ der Raum jener Funktionen aus $\mathscr{E}(\Omega)$, die ihren Träger in $K$ haben. Der Raum $\mathscr{D}_{K}(\Omega)$ werde mit der von $\mathscr{E}(\Omega)$ induzierten Topologie versehen. Ferner sei $\mathscr{D}(\Omega)$ der Raum der unendlich oft differenzierbaren Funktionen mit kompaktem Träger, versehen mit der Topologie des induktiven Limes der Räume $\mathscr{D}_{K}(\Omega)$.

Die Räume $\mathscr{E}^{\prime}(\Omega)$ und $\mathscr{D}^{\prime}(\Omega)$ der linearen und stetigen Funktionale auf $\mathscr{E}(\Omega)$ und $\mathscr{D}(\Omega)$ werden mit der starken Topologie versehen. $\mathscr{D}^{\prime}(\Omega)$ heißt Raum der Distributionen in $\Omega$. Der Raum $\mathscr{E}^{\prime}(\Omega)$ kann mit dem Unterraum jener Distributionen aus $\mathscr{D}^{\prime}(\Omega)$ identifiziert werden, die kompakte Träger in $\Omega$ haben. Schließlich kann der Raum $L_{\mathrm{loc}}^{1}(\Omega)$ der Äquivalenzklassen meßbarer und lokalintegrabler Funktionen mit einem Teilraum von $\mathscr{D}^{\prime}(\Omega)$ identifiziert werden. Die Elemente von $\mathscr{D}^{\prime}(\Omega)$ werden gelegentlich auch als verallgemeinerte Funktionen bezeichnet, so daß symbolisch

$$
\{\text { Funktionen }\}=L_{\mathrm{loc}}^{1}(\Omega) \subset \mathscr{D}^{\prime}(\Omega)=\{\text { verallgemeinerte Funktionen }\}
$$

geschrieben werden kann. Den Raum der Äquivalenzklassen meßbarer und quadratintegrabler Funktionen bezeichnen wir mit $L^{2}(\Omega)$. Es gilt $L^{2}(\Omega) \subset L_{\mathrm{loc}}^{1}(\Omega)$. Die Norm in $L^{2}(\Omega)$ wird mit \|\|$_{2}$, die Norm in $L^{1}(\Omega)$ mit \|\|$_{1}$ bezeichnet.

Für $\Omega=\boldsymbol{R}^{n}$ wird $\mathscr{E}\left(\boldsymbol{R}^{n}\right)=\mathscr{E}, \mathscr{D}\left(\boldsymbol{R}^{n}\right)=\mathscr{D}, L^{2}\left(\boldsymbol{R}^{n}\right)=L^{2}$, usw. geschrieben.

Für die Fouriertransformation benötigen wir noch den Raum $\mathscr{S}$ der unendlich oft differenzierbaren Funktionen, die samt allen ihren Ableitungen schneller als der Reziprokwert jedes Polynoms im Unendlichen gegen 0 gehen, versehen mit der üblichen Topologie [15], S. 92. Dann ist $\mathscr{S}^{\prime}$ der Raum der temperierten Distributionen, der ebenfalls mit einem Unterraum von $\mathscr{D}^{\prime}$ identifiziert werden kann. Wir haben dann $\mathscr{D} \subset \mathscr{S} \subset \mathscr{E}$ und $\mathscr{E}^{\prime} \subset \mathscr{S}^{\prime} \subset \mathscr{D}^{\prime}$.

1.3. Die für den Beweis des Malgrange--Ehrenpreis-Theorems grundlegende Ungleichung

$$
\left\|P^{(p)}(\partial) \varphi\right\|_{2} \leqq C\left\|e^{\varepsilon\|x\|} P(\partial) \varphi\right\|_{2}
$$

wurde in Schwartz [12] nur zitiert. Sie findet sich für $L^{2}(\Omega)$ mit beschränktem $\Omega$ und daher ohne den Faktor $e^{\varepsilon\|x\|}$ zum ersten Mal in der Dissertation von L. Hörmander [3], S. 183-185. Im folgenden wird diese Ungleichung nach einer Idee von F. Treves [14], der den ursprünglichen Hörmanderschen Beweis modifizierte, in $\boldsymbol{R}^{n}$ bewiesen, wozu nach Hörmander die Einführung des Faktors $e^{\varepsilon\|x\|}$ nötig ist. Für wertvolle Hinweise danke ich an dieser Stelle Herrn I. S. Louhivaara. 


\section{Partielle Differentialgleichungen mit konstanten Koeffizienten und Fundamentallösungen}

Sei $P$ ein Polynom der Ordnung $m$ auf $\boldsymbol{R}^{n}$,

$$
P: R^{n} \rightarrow C, \quad \xi \mapsto \sum_{|j| \leqq m} a_{j} \xi^{j},
$$

mit komplexen Koeffizienten $a_{j} \in C$ und $m \in N_{0}\left(j=\left(j_{1}, \ldots, j_{n}\right) \in N_{0}^{n},|j|=j_{1}+\ldots+j_{n}\right)$. Ferner sei angenommen, daß mindestens ein $a_{j}$ mit $|j|=m$ von Null verschieden ist. Ersetzt man $\xi$ durch $\partial=\partial / \partial x=\left(\partial / \partial x_{1}, \ldots, \partial / \partial x_{n}\right)$ und schreibt man

$$
\partial^{j}=\left(\frac{\partial}{\partial x}\right)^{j}=\frac{\partial^{|j|}}{\partial x^{j}}:=\frac{\partial^{|j|}}{\partial x_{1}^{j_{1}} \ldots \partial x_{n}^{j_{n}}},
$$

so ergibt sich danach

eine Abbildung

$$
P(\partial) \varphi=\sum_{|j| \leqq m} a_{j} \frac{\partial^{|j|} \varphi}{\partial x^{j}} \quad \text { für } \quad \varphi \in \mathscr{D}
$$

$$
P(\partial): \mathscr{D} \rightarrow \mathscr{D}, \quad \varphi \mapsto P(\partial) \varphi,
$$

die man einen linearen partiellen Differentialoperator (mit konstanten Koeffizienten) nennt. Die Abbildung

$$
P^{\smile}(\partial): \mathscr{D} \rightarrow \mathscr{D}, \quad \varphi \mapsto \sum_{|j| \leqq m} a_{j}(-1)^{|j|} \frac{\partial^{|j|} \varphi}{\partial x^{j}}
$$

heißt der dem Differentialoperator $P(\partial)$ adjungierte Differentialoperator.

Jeder Differentialoperator ist eine lineare und stetige Abbildung von $\mathscr{D}$ in $\mathscr{D}$. Also existiert die Transponierte ${ }^{t} P^{\smile}(\partial)$ als lineare und stetige Abbildung von $\mathscr{D}^{\prime}$ in $\mathscr{D}^{\prime}$. Sie heißt der dem Differentialoperator $P(\partial)$ entsprechende lineare partielle Differentialoperator in $\mathscr{D}^{\prime}$ und wird wieder mit $P(\partial)$ bezeichnet. Diese Bezeichnungsweise findet ihre Begründung in folgendem Konsistenzsatz: Ist $f \in C^{m}$ und $T_{f}$ die zugeordnete Distribution, so gilt:

$$
P(\partial) T_{f}=T_{g} \quad \text { mit } \quad g:=P(\partial) f:=\sum_{|j| \leqq m} a_{j} \partial^{j} f .
$$

(Wegen $C^{m} \subset L_{\text {loc }}^{1} \subset \mathscr{D}^{\prime}$ wird in Hinkunft zwischen $f$ und $T_{f}$ nicht mehr unterschieden.)

Ist $T \in \mathscr{D}^{\prime}$, so heißt jedes $S \in \mathscr{D}^{\prime}$ mit

$$
P(\partial) S=T,
$$

eine Lösung der Differentialgleichung

$$
P(\partial) X=T \text {. }
$$

Sei $\delta$ die Diracsche Distribution. Eine Lösung der Differentialgleichung

$$
P(\partial) X=\delta
$$

heißt Fundamentallösung des Differentialoperators $P(\partial)$. 
Satz 1. Sei $T \in \mathscr{E}^{\prime}$ und E eine Fundamentallösung des Differentialoperators $P(\partial)$. Dann ist die Faltung $E * T$ eine Lösung der Differentialgleichung

$$
P(\partial) X=T \text {. }
$$

Außerdem hängt $E * T$ stetig von $T$ ab (im Sinne der Topologien von $\mathscr{D}^{\prime}$ und $\mathscr{E}^{\prime}$ ).

Beweis. Nach [15], S. 294, Satz 27.3, und S. 295, Satz 27.5, ist

$$
P(\partial)(E * T)=\sum_{|j| \leqq m} a_{j} \partial^{j}(E * T)=\left(\sum_{|j| \leqq m} a_{j} \partial^{j} E\right) * T=\delta * T=T .
$$

Die stetige Abhängigkeit folgt unmittelbar aus [15], S. 294, Theorem 27.6.

Bemerkungen. (1) Wenn $T$ einen kompakten Träger hat, so reduziert sich also das Lösen der Differentialgleichung

$$
P(\partial) X=T
$$

auf das Aufsuchen einer Fundamentallösung des Differentialoperators $P(\partial)$.

(2) Die Lösung der Differentialgleichung ist keineswegs eindeutig: Für $\lambda \in C^{n}$ mit $P(\lambda)=0$ ist

$$
\sum_{|j| \leqq m} a_{j} \partial^{j}\left(e^{\lambda x}\right)=\left(\sum_{|j| \leqq m} a_{j} \lambda^{j}\right) e^{\lambda x}=P(\lambda) e^{\lambda x}=0
$$

und wegen der Linearität von $P(\partial)$

$$
P(\partial)\left(E * T+e^{\lambda x}\right)=T,
$$

so daß neben $E * T$ auch $E * T+e^{\lambda x}$ eine Lösung ist.

(3) Ist $E$ eine Fundamentallösung von $P(\partial)$ und ist $f \in \mathscr{D}$, so liegt die Faltung $f_{*} E$ nach [15], S. 293, Theorem 27.5, in $\mathscr{E}$, und ist eine Lösung der Differentialgleichung

$$
P(\partial) X=f .
$$

\section{Einfache Beispiele von Fundamentallösungen}

3.1. Sei $n=1$. Die durch

$$
Y_{a}: R \rightarrow C, \quad x \mapsto\left\{\begin{array}{lll}
1 & \text { für } & x \geqq a \\
0 & \text { für } x<a,
\end{array} \text { mit } a \in \boldsymbol{R}\right.
$$

definierte Funktion $Y_{a} \in L_{\text {loc }}^{1}$ heißt Heavisidefunktion. Nach [15], S. 251, ist dann

$$
\frac{d}{d x} Y_{a}=\delta_{a},
$$

das heißt $Y:=Y_{0}$ ist eine Fundamentallösung des gewöhnlichen Differentialoperators $d / d x$ (in $\mathscr{D}^{\prime}$ ). 
3.2. Sei $n=2$. Ferner sei

$$
Y_{a}: R^{2} \rightarrow C, \quad(x, y) \mapsto\left\{\begin{array}{ll}
1 & \text { für } x \geqq \alpha, \quad y \geqq \beta \\
0 & \text { sonst, }
\end{array} \text { mit } a=(\alpha, \beta) \in \boldsymbol{R}^{2} .\right.
$$

Dann wird für $\varphi \in \mathscr{D}$

$$
\left\langle\varphi, \partial_{x y}^{2} Y_{a}\right\rangle=\left\langle\partial_{x y}^{2} \varphi, Y_{a}\right\rangle=\int_{\alpha}^{\infty} \int_{\beta}^{\infty} \partial_{x y}^{2} \varphi d x d y=\varphi(\alpha, \beta)=\left\langle\varphi, \delta_{a}\right\rangle
$$

und folglich

$$
\partial_{x y}^{2} Y_{a}=\delta_{a},
$$

das heißt $Y:=Y_{0}$ ist eine Fundamentallösung des Differentialoperators $\partial_{x y}^{2}$.

Aus diesem Beispiel läßt sich unschwer eine Fundamentallösung des Wellenoperators im $\boldsymbol{R}^{2}$ vermuten:

3.3. Sei $n=2$ und

Dann wird für $\varphi \in \mathscr{D}$

$$
Y^{\sim}: \mathbb{R}^{2} \rightarrow C, \quad(x, y) \mapsto \begin{cases}1 & \text { wenn } \quad x \geqq|y| \\ 0 & \text { sonst. }\end{cases}
$$

$$
\begin{gathered}
\left\langle\varphi, \partial_{x}^{2} Y^{\sim}-\partial_{y}^{2} Y^{\sim}\right\rangle=\left\langle\partial_{x}^{2} \varphi-\partial_{y}^{2} \varphi, Y^{\sim}\right\rangle=\iint_{x \geqq|y|} d x d y\left(\partial_{x}^{2} \varphi-\partial_{y}^{2} \varphi\right) \\
=\int_{-\infty}^{0} d y \int_{-y}^{\infty} d x \partial_{x}^{2} \varphi+\int_{0}^{\infty} d y \int_{y}^{\infty} d x \partial_{x}^{2} \varphi-\int_{0}^{\infty} d x \int_{-x}^{x} d y \partial_{y}^{2} \varphi \\
=-\int_{-\infty}^{0} d y \partial_{x} \varphi(-y, y)-\int_{0}^{\infty} d y \partial_{x} \varphi(y, y) \\
-\int_{0}^{\infty} d x\left(\partial_{y} \varphi(x, x)-\partial_{y} \varphi(x,-x)\right) \\
=-\int_{0}^{\infty} d t\left(\partial_{x} \varphi-\partial_{y} \varphi\right)(t,-t)-\int_{0}^{\infty} d t\left(\partial_{x} \varphi+\partial_{y} \varphi\right)(t, t) .
\end{gathered}
$$

Mit $\Phi(t):=\varphi(t, t)$ und $\Psi(t):=\varphi(t,-t)$ ist

$$
\frac{d \Phi}{d t}(t)=\left(\partial_{x} \varphi+\partial_{y} \varphi\right)(t, t) \text { und } \frac{d \Psi}{d t}(t)=\left(\partial_{x} \varphi-\partial_{y} \varphi\right)(t,-t)
$$

Somit ergibt sich

$$
\begin{aligned}
\left\langle\varphi, \partial_{x}^{2} Y^{\sim}-\partial_{y}^{2} Y^{\sim}\right\rangle & =-\int_{0}^{\infty} \frac{d \Psi}{d t} d t-\int_{0}^{\infty} \frac{d \Phi}{d t} d t=\Psi(0)+\Phi(0) \\
& =2 \varphi(0,0)=\langle\varphi, 2 \delta\rangle .
\end{aligned}
$$

Also ist $(1 / 2) Y^{\sim}$ eine Fundamentallösung des Wellenoperators $\partial_{x}^{2}-\partial_{y}^{2}$. 
3.4. Sei wieder $n=2$, und sei

$s: \boldsymbol{R}^{2} \rightarrow C$ durch $s(x, y):=\left\{\begin{array}{lll}\frac{1}{\pi} \frac{1}{x+i y} & \text { für } & (x, y) \neq(0,0) \\ 0 & \text { für } & (x, y)=(0,0)\end{array}\right.$

definiert. Es gilt $s \in L_{\text {loc }}^{1}$ Zum Beweis benötigt man folgenden Hilfssatz aus der Integrationstheorie: Für eine positive und stetige Funktion $F:(0, \infty) \rightarrow R$ sind die beiden Aussagen äquivalent:

(1) Die Abbildung $\boldsymbol{R}^{n} \rightarrow \boldsymbol{R}, x \mapsto\left\{\begin{array}{ll}F(\|x\|) & \text { für } x \neq 0 \\ 0 & \text { für } x=0\end{array}\right.$ ist in $\boldsymbol{R}^{n}$ integrierbar.

(2) Das uneigentliche Riemannintegral $\int_{0}^{\infty} F(t) t^{n-1} d t$ existiert.

Erfüllt $F$ die Aussagen, so gilt zusätzlich

$$
\int F(\|x\|) d x=\omega_{n} \int_{0}^{\infty} F(t) t^{n-1} d t
$$

wobei $\omega_{n}$ der Oberflächeninhalt der $n$-dimensionalen Einheitskugel ist. Zum Beweis vergleiche man Schwartz [13], S. 39.

Wir zeigen nun, daß $s$ eine Fundamentallösung des Cauchy-Riemannoperators $(1 / 2)\left(\partial_{x}+i \partial_{y}\right)$ ist.

Für $\varphi \in \mathscr{D}$ wird

$$
\begin{aligned}
I:=\left\langle\varphi,(1 / 2)\left(\partial_{x} s+i \partial_{y} s\right)\right\rangle & =(-1 / 2)\left\langle\partial_{x} \varphi+i \partial_{y} \varphi, s\right\rangle \\
& =-\frac{1}{2} \lim _{m \rightarrow \infty} \int_{\|x\| \geqq 1 / m} s\left(\partial_{x} \varphi+i \partial_{y} \varphi\right) d x .
\end{aligned}
$$

Nach Einführung von Polarkoordinaten folgt

$$
I=-\frac{1}{2} \lim _{m \rightarrow \infty} \int_{1 / m}^{\infty} r d r \int_{0}^{2 \pi} d \vartheta s(r \cos \vartheta, r \sin \vartheta)\left(\partial_{x} \varphi+i \partial_{y} \varphi\right)(r \cos \vartheta, r \sin \vartheta) .
$$

Es ist

$$
s(r \cos \vartheta, r \sin \vartheta)=\frac{1}{\pi r} e^{-i \vartheta} \quad(r \neq 0) .
$$

Mit $\Phi(r, \vartheta):=\varphi(r \cos \vartheta, r \sin \vartheta)$ wird

$$
\left(\partial_{r} \Phi+(i / r) \partial_{\vartheta} \Phi\right)(r, \vartheta)=e^{-i \vartheta}\left(\partial_{x} \varphi+i \partial_{y} \varphi\right)(r \cos \vartheta, r \sin \vartheta)
$$


und

$$
\begin{aligned}
I & =-\frac{1}{2 \pi} \lim _{m \rightarrow \infty} \int_{1 / m}^{\infty} d r \int_{0}^{2 \pi} d \vartheta\left(\partial_{r} \Phi+(i / r) \partial_{\vartheta} \Phi\right) \\
& =-\frac{1}{2 \pi} \lim _{m \rightarrow \infty}\left(\int_{0}^{2 \pi} d \vartheta \int_{1 / m}^{\infty} d r \partial_{r} \Phi+i \int_{1 / m}^{\infty} d r / r \int_{0}^{2 \pi} d \vartheta \partial_{\vartheta} \Phi\right) \\
& =\frac{1}{2 \pi} \lim _{m \rightarrow \infty}\left(\int_{0}^{2 \pi} \varphi((1 / m) \cos \vartheta,(1 / m) \sin \vartheta) d \vartheta-i \int_{1 / m}^{\infty}(d r / r)(\Phi(r, 2 \pi)-\Phi(r, 0))\right) \\
& =\frac{1}{2 \pi} \int_{0}^{2 \pi} \varphi(0,0) d \vartheta-\frac{i}{2 \pi} \lim _{m \rightarrow \infty} \int_{1 / m}^{\infty}(d r / r)(\varphi(r, 0)-\varphi(r, 0))=\varphi(0,0)=\langle\varphi, \delta\rangle .
\end{aligned}
$$

Schließlich folgt

$$
\frac{1}{2}\left(\partial_{x} s+i \partial_{y} s\right)=\delta
$$

\section{Gewinnung einer Fundamentallösung durch Fouriertransformation}

In den angeführten Beispielen haben wir Fundamentallösungen einiger Differentialoperatoren gefunden durch Anwendung dieser Differentialoperatoren auf speziell gewählte Distributionen. Selbstverständlich ist dieses Verfahren zur Gewinnung von Fundamentallösungen nur sehr beschränkt brauchbar. Wir stellen uns deshalb die Frage: Wie können Fundamentallösungen systematisch konstruiert werden?

4.1. Wir wollen eine Fundamentallösung des Laplaceoperators durch Fouriertransformation in $\mathscr{S}^{\prime}$ ermitteln.

Zunächst nehmen wir an, es gebe $E_{\varepsilon} \in \mathscr{S}^{\prime}$ mit

$$
4 \pi^{2} \varepsilon^{2} E_{\varepsilon}-\Delta_{n} E_{\varepsilon}=\delta
$$

für $\varepsilon>0$. Dann folgt aus [15], S. 277, Theorem 25.7,

$$
4 \pi^{2} \varepsilon^{2} \mathscr{F} E_{\varepsilon}-(2 \pi i)^{2}\left(\sum_{j=1}^{n} \xi_{j}^{2}\right) \mathscr{F} E_{\varepsilon}=4 \pi^{2}\left(\varepsilon^{2}+\|\xi\|^{2}\right) \mathscr{F} E_{\varepsilon}=1,
$$

also gilt für jedes $\psi \in \mathscr{S}$

Mit $\varphi \in \mathscr{S}$ ist

$$
\left\langle 4 \pi^{2}\left(\varepsilon^{2}+\|\xi\|^{2}\right) \psi, \mathscr{F} E_{\varepsilon}\right\rangle=\int \psi d \xi .
$$

$$
\frac{1}{\varepsilon^{2}+\|\xi\|^{2}} \varphi \in \mathscr{S}
$$

da

$$
\frac{1}{\varepsilon^{2}+\|\xi\|^{2}} \in \mathcal{O}_{M}
$$


gilt; $\mathcal{O}_{M}$ ist der Raum der Funktionen aus $\mathscr{E}$, die mit allen Ableitungen im Unendlichen langsam wachsen (vgl. [15], S. 275). Für

ergibt sich

$$
\psi:=\frac{1}{\varepsilon^{2}+\|\xi\|^{2}} \varphi \in \mathscr{S}
$$

Mit

$$
\left\langle\varphi, \mathscr{F} E_{\varepsilon}\right\rangle=\frac{1}{4 \pi^{2}} \int \frac{1}{\varepsilon^{2}+\|\xi\|^{2}} \varphi d \xi
$$

können wir auch schreiben

$$
f_{\varepsilon}(\xi):=\frac{1}{4 \pi^{2}\left(\varepsilon^{2}+\|\xi\|^{2}\right)}
$$

oder

$$
\left\langle\varphi, \mathscr{F} E_{\varepsilon}\right\rangle=\left\langle\varphi, f_{\varepsilon}\right\rangle
$$

$$
\mathscr{F} E_{\varepsilon}=f_{\varepsilon} \quad \text { und } \quad \overline{\mathscr{F}} \mathscr{F} E_{\varepsilon}=\overline{\mathscr{F}} f_{\varepsilon}
$$

Nach [15], S. 276, Theorem 25.6, ist $\mathscr{F}$ ein Isomorphismus in $\mathscr{S}^{\prime}$ und wir haben

$$
E_{\varepsilon}=\overline{\mathscr{F}} f_{\varepsilon} \text {. }
$$

Sei nun umgekehrt $E_{\varepsilon}$ durch diese Gleichung definiert. Dann ist $E_{\varepsilon} \in \mathscr{S}^{\prime}$, weil $f_{\varepsilon} \in \mathscr{S}^{\prime}$ gilt. Weiter wird nach [15], S. 277, Theorem 25.7,

$$
4 \pi^{2} \varepsilon^{2} \overline{\mathscr{F}} f_{\varepsilon}-\Delta_{n}\left(\overline{\mathscr{F}} f_{\varepsilon}\right)=\overline{\mathscr{F}}\left(4 \pi^{2} \varepsilon^{2} f_{\varepsilon}+4 \pi^{2} \xi^{2} f_{\varepsilon}\right)=\overline{\mathscr{F}}(1)=\delta,
$$

das heißt $E_{\varepsilon}$ ist die Fundamentallösung in $\mathscr{S}^{\prime}$ von $4 \pi^{2} \varepsilon^{2}-\Delta_{n}$.

4.2. Als nächstes soll $f_{\varepsilon}$ berechnet werden:

Für $n=1$ ist $f_{\varepsilon} \in L^{1}$. Also gilt

$$
\overline{\mathscr{F}} f_{\varepsilon}(x)=\frac{1}{4 \pi^{2}} \int e^{2 \pi i x \xi} \frac{d \xi}{\varepsilon^{2}+\xi^{2}}
$$

Nach [1], S. 135, (13.9.16) und [2], S. 406, Formel 3.723/6, ist

$$
\overline{\mathscr{F}} f_{\varepsilon}(x)=\frac{1}{4 \pi^{2}} \int_{-\infty}^{\infty} e^{2 \pi i x \xi} \frac{d \xi}{\varepsilon^{2}+\xi^{2}}=\frac{1}{4 \pi \varepsilon} e^{-2 \pi \varepsilon|x|}
$$

Für $n \geqq 2$ sei $x^{\prime}:=\left(x_{1}, \ldots, x_{n-1}\right), d x^{\prime}:=d x_{1} \ldots d x_{n-1}$ und $\left\|x^{\prime}\right\|:=\left(\sum_{j=1}^{n-1} x_{j}^{2}\right)^{1 / 2}$. Mit $\varphi \in \mathscr{S}$ wird

$$
\left\langle\varphi, \overline{\mathscr{F}} f_{\varepsilon}\right\rangle=\int f_{\varepsilon} \overline{\mathscr{F}} \varphi d \xi=\frac{1}{4 \pi^{2}} \int \frac{d \xi}{\varepsilon^{2}+\|\xi\|^{2}} \overline{\mathscr{F}} \varphi(\xi) .
$$

Durch zweimalige Anwendung von [1], S. 203, (13.21.7), folgt

$$
\left\langle\varphi, \overline{\mathscr{F}} f_{\varepsilon}\right\rangle=\frac{1}{4 \pi^{2}} \int d \xi^{\prime} \int \frac{d \xi_{n}}{\varepsilon^{2}+\left\|\xi^{\prime}\right\|^{2}+\xi_{n}^{2}} \int d x_{n} \int d x^{\prime} e^{2 \pi i x \xi} \varphi(x) .
$$


Da die Funktion

$$
x_{n} \mapsto \int d x^{\prime} e^{2 \pi i x \xi} \varphi(x)
$$

in $L^{1}\left(\boldsymbol{R}^{1}\right)$ ist, gilt unter Berücksichtigung von [1], S. 203, (13.21.7), und S. 204, (13.21.9),

$$
\left\langle\varphi, \overline{\mathscr{F}} f_{\varepsilon}\right\rangle=\frac{1}{4 \pi^{2}} \int d \xi^{\prime} \int d x_{n} \int \frac{d \xi_{n}}{\varepsilon^{2}+\left\|\xi^{\prime}\right\|^{2}+\xi_{n}^{2}} e^{2 \pi i x_{n} \xi_{n}} \int d x^{\prime} e^{2 \pi i x^{\prime} \xi^{\prime}} \varphi(x) .
$$

Wegen

$$
\int_{-\infty}^{\infty} \frac{e^{2 \pi i x_{n} t}}{t^{2}+\varepsilon^{2}+\left\|\xi^{\prime}\right\|^{2}} d t=\frac{\pi}{\sqrt{\varepsilon^{2}+\left\|\xi^{\prime}\right\|^{2}}} e^{-2 \pi\left|x_{n}\right| \sqrt{\varepsilon^{2}+\left\|\xi^{\prime}\right\|^{2}}}
$$

gilt nach [1], S. 135, (13.9.16),

$$
\begin{aligned}
\left\langle\varphi, \overline{\mathscr{F}} f_{\varepsilon}\right\rangle & =\frac{1}{4 \pi} \int d \xi^{\prime} \int d x_{n} \frac{e^{-2 \pi\left|x_{n}\right| \sqrt{\varepsilon^{2}+\left\|\xi^{\prime}\right\|^{2}}}}{\sqrt{\varepsilon^{2}+\left\|\xi^{\prime}\right\|^{2}}} \int d x^{\prime} e^{2 \pi i x^{\prime} \xi^{\prime}} \varphi(x) \\
& =\frac{1}{4 \pi} \int d \xi^{\prime} \int d x_{n} \frac{e^{-2 \pi\left|x_{n}\right| \sqrt{\varepsilon^{2}+\left\|\xi^{\prime}\right\|^{2}}}}{\left(\varepsilon^{2}+\left\|\xi^{\prime}\right\|^{2}\right)^{1 / 2+k}}\left(\varepsilon^{2}+\left\|\xi^{\prime}\right\|^{2}\right)^{k} \int d x^{\prime} e^{2 \pi i x^{\prime} \xi^{\prime}} \varphi(x) ;
\end{aligned}
$$

hierbei ist

$$
\left(\varepsilon^{2}+\left\|\xi^{\prime}\right\|^{2}\right)^{k} \int d x^{\prime} e^{2 \pi i x^{\prime} \xi^{\prime}} \varphi(x) \in \mathscr{S} \subset L^{\infty} .
$$

Daher konvergiert für genügend große $k$ (etwa $k=[n / 2]+1$; vgl. den Beweis von Satz 3) das iterierte Integral absolut. Wegen [1], S. 204, (13.21.9), und S. 203, (13.21.7), ergibt sich

$$
\left\langle\varphi, \overline{\mathscr{F}} f_{\varepsilon}\right\rangle=\frac{1}{4 \pi} \int d x_{n} \int d \xi^{\prime} \frac{e^{-2 \pi\left|x_{n}\right| \sqrt{\varepsilon^{2}+\left\|\xi^{\prime}\right\|^{2}}}}{\sqrt{\varepsilon^{2}+\left\|\xi^{\prime}\right\|^{2}}} \int d x^{\prime} e^{2 \pi i x^{\prime} \zeta^{\prime}} \varphi(x),
$$

und für $x_{n} \neq 0$ sind weiters die Integrationen nach $\xi^{\prime}$ und $x^{\prime}$ vertauschbar:

$$
\left\langle\varphi, \overline{\mathscr{F}} f_{\varepsilon}\right\rangle=\frac{1}{4 \pi} \int d x_{n} \int d x^{\prime} \varphi(x) \int d \xi^{\prime} \frac{e^{-2 \pi\left|x_{n}\right| \sqrt{\varepsilon^{2}+\left\|\xi^{\prime}\right\|^{2}}+2 \pi i x^{\prime} \xi^{\prime}}}{\sqrt{\varepsilon^{2}+\left\|\xi^{\prime}\right\|^{2}}} .
$$

Das Integral

$$
J:=\int d \xi^{\prime} \frac{e^{-2 \pi\left|x_{n}\right| \sqrt{\varepsilon^{2}+\left\|\xi^{\prime}\right\|^{2}}+2 \pi i x^{\prime} \xi^{\prime}}}{\sqrt{\varepsilon^{2}+\left\|\xi^{\prime}\right\|^{2}}}
$$

existiert für $x_{n} \neq 0$. Sei $R$ eine Drehung des Raumes $\boldsymbol{R}_{\xi^{\prime}}^{n-1}$, die den Vektor $x^{\prime}$ in die $\eta_{n-1}$-Achse des gedrehten Koordinatensystems $\eta_{1}, \ldots, \eta_{n-1}$ überführt. Dann ist $\left\|\xi^{\prime}\right\|=\left\|\eta^{\prime}\right\|$ und $\xi^{\prime} x^{\prime}=\eta_{n-1}\left\|x^{\prime}\right\|$. Außerdem ist $R$ ein Homöomorphismus in $\boldsymbol{R}^{n-1}$, und das Bild des Lebesguemaßes $d \xi^{\prime}$ unter $R$ ist $d \eta^{\prime}$. Nach [1], S. 122, (13.7.10), ist daher

$$
J=\int d \eta^{\prime} \frac{e^{-2 \pi\left|x_{n}\right| \sqrt{\varepsilon^{2}+\left\|\eta^{\prime}\right\|^{2}}+2 \pi i \eta_{n-1}\left\|x^{\prime}\right\|}}{\sqrt{\varepsilon^{2}+\left\|\eta^{\prime}\right\|^{2}}} .
$$


Für $n=2$ ist nach [1], S. 135, (13.9.16), und [2], S. 498, Formel 3.961, 2,

$$
J=2 K_{0}(2 \pi \varepsilon\|x\|),
$$

wobei $K_{\tau}$ die modifizierte Besselfunktion der Ordnung $\tau$ ist.

Für $n>2$ sei analog $\eta^{\prime \prime}:=\left(\eta_{1}, \ldots, \eta_{n-2}\right), d \eta^{\prime \prime}:=d \eta_{1} \ldots d \eta_{n-2}$ und $\left\|\eta^{\prime \prime}\right\|:=\left(\sum_{j=1}^{n-2} \eta_{j}^{2}\right)^{1 / 2}$. Folglich gilt

$$
\begin{aligned}
J & =\int d \eta^{\prime \prime} \int d \eta_{n-1} \frac{e^{-2 \pi\left|x_{n}\right| \sqrt{\varepsilon^{2}+\left\|\eta^{\prime \prime}\right\|^{2}+\eta_{n-1}^{2}}}}{\sqrt{\varepsilon^{2}+\left\|\eta^{\prime \prime}\right\|^{2}+\eta_{n-1}^{2}}} e^{2 \pi i \eta_{n-1}\left\|x^{\prime}\right\|} \\
& =2 \int d \eta^{\prime \prime} K_{0}\left(2 \pi\|x\| \sqrt{\varepsilon^{2}+\left\|\eta^{\prime \prime}\right\|^{2}}\right)
\end{aligned}
$$

Da die unter dem Integralzeichen stehende Funktion stetig und positiv ist (wegen $\varepsilon>0$ und $x_{n} \neq 0$ ), ist nach dem Hilfssatz aus 3.4

$$
J=2 \omega_{n-2} \int_{0}^{\infty} \varrho^{n-3} K_{0}\left(2 \pi\|x\| \sqrt{\varepsilon^{2}+\varrho^{2}}\right) d \varrho
$$

und nach [2], S. 705, Formel 6.596, 3,

Somit haben wir

$$
\begin{aligned}
J & =2 \omega_{n-2} \frac{2^{n / 2-2} \Gamma(n / 2-1)}{(2 \pi\|x\|)^{n / 2-1} \varepsilon^{-n / 2+1}} K_{-n / 2+1}(2 \pi \varepsilon\|x\|) \\
& =2\left(\frac{\varepsilon}{\|x\|}\right)^{n / 2-1} K_{n / 2-1}(2 \pi \varepsilon\|x\|) .
\end{aligned}
$$

$$
\left\langle\varphi, \overline{\mathscr{F}} f_{\varepsilon}\right\rangle=\frac{1}{2 \pi} \int d x_{n} \int d x^{\prime} \varphi(x)\left(\frac{\varepsilon}{\|x\|}\right)^{n / 2-1} K_{n / 2-1}(2 \pi \varepsilon\|x\|) .
$$

4.3. Mit den in 4.1 und 4.2 gewonnenen Funktionen definieren wir für $n \quad$ l

$$
k_{\varepsilon}(x):=\frac{1}{4 \pi \varepsilon} e^{-2 \pi \varepsilon|x|}
$$

und für $n \geqq 2$

$$
k_{\varepsilon}(x):=\left\{\begin{array}{lll}
\frac{1}{2 \pi}\left(\frac{\varepsilon}{\|x\|}\right)^{n / 2-1} K_{n / 2-1}(2 \pi \varepsilon\|x\|) & \text { für } & x \neq 0 \\
0 & \text { für } & x=0 .
\end{array}\right.
$$

Im Fall $n=1$ ist $k_{\varepsilon} \in L^{1}$ und $\overline{\mathscr{F}} f_{\varepsilon}=k_{\varepsilon}$.

Also ist

$$
\frac{1}{4 \pi \varepsilon} e^{-2 \pi \varepsilon|x|}
$$

eine Fundamentallösung von

$$
4 \pi^{2} \varepsilon^{2}-\frac{d^{2}}{d x^{2}}
$$

in $\mathscr{S}^{\prime}$. 
Wir werden zeigen, daß $k_{\varepsilon} \in L^{1}$ auch für $n \geqq 2$ gilt. Dazu wenden wir wieder den Hilfssatz aus 3.4 an. Nach [2], S. 958, Formel 8.432, 3, ist

$$
\begin{gathered}
\left(\frac{\varepsilon}{\|x\|}\right)^{n / 2-1} K_{n / 2-1}(2 \pi \varepsilon\|x\|)= \\
=\left(\frac{\varepsilon}{\|x\|}\right)^{n / 2-1} \frac{\Gamma(1 / 2)}{\Gamma(n / 2-1)}(\pi \varepsilon\|x\|)^{n / 2-1} \int_{1}^{\infty} e^{-2 \pi \varepsilon\|x\| t}\left(t^{2}-1\right)^{(n-3) / 2} d t \\
=\frac{\Gamma(1 / 2)}{\Gamma((n-1) / 2)} \pi^{n / 2-1} \varepsilon^{n-2} \int_{1}^{\infty} e^{-2 \pi \varepsilon\|x\| t}\left(t^{2}-1\right)^{(n-3) / 2} d t,
\end{gathered}
$$

also gilt $k_{\varepsilon}(x) \geqq 0$. Weiters ist ([2], S. 684 , Formel $\left.6.561,16\right)$

$$
\begin{aligned}
& \frac{1}{\pi} \int_{0}^{\infty} \frac{\varepsilon^{n / 2-1}}{t^{n / 2-1}} t^{n-1} K_{n / 2-1}(2 \pi \varepsilon t) d t=\frac{1}{4 \pi^{2} \varepsilon^{2} \pi^{n / 2} 2^{n / 2}} \int_{0}^{\infty} \varrho^{n / 2} K_{n / 2-1}(\varrho) d \varrho \\
& =\frac{1}{4 \pi^{2} \varepsilon^{2} \pi^{n / 2} 2^{n / 2}} 2^{n / 2-1} \Gamma\left(\frac{1+n / 2+n / 2-1}{2}\right) \Gamma\left(\frac{1+n / 2-n / 2+1}{2}\right) \\
& \quad=\frac{\Gamma(n / 2) 2^{n / 2-1}}{4 \pi^{2} \varepsilon^{2} \pi^{n / 2} 2^{n / 2}}=\frac{\Gamma(n / 2)}{8 \varepsilon^{2} \pi^{n / 2+2}} .
\end{aligned}
$$

Also folgt aus dem Hilfssatz in 3.4

$$
\int k_{\varepsilon} d x=\frac{\omega_{n} \Gamma(n / 2)}{8 \varepsilon^{2} \pi^{n / 2+2}}-\frac{1}{4 \pi^{2} \varepsilon^{2}}
$$

womit $k_{\varepsilon} \in L^{1}$ gezeigt ist.

Daher ist $k_{\varepsilon}$ auch für $n \geqq 2$ eine Fundamentallösung des Differentialoperators

in $\mathscr{S}^{\prime}$.

$$
4 \pi^{2} \varepsilon^{2}-\Delta_{n}
$$

Für $n=2$ ist

$$
k_{\varepsilon}(x)=\frac{1}{2 \pi} K_{0}(2 \pi \varepsilon\|x\|) \quad(x \neq 0)
$$

und für $n=3$ folgt aus der obigen Integraldarstellung

$$
k_{\varepsilon}(x)=\frac{1}{2 \pi} \Gamma(1 / 2) \pi^{1 / 2} \varepsilon \int_{1}^{\infty} e^{-2 \pi \varepsilon\|x\| t} d t=\frac{e^{-2 \pi \varepsilon\|x\|}}{4 \pi\|x\|} \quad(x \neq 0) .
$$


4.4. Als nächstes geben wir eine Abschätzung für $k_{\varepsilon}$ im Fall $n \geqq 3$ an. Aus der Integraldarstellung folgt $(x \neq 0)$

$$
\begin{aligned}
k_{\varepsilon}(x) & =\frac{\pi^{(n-3) / 2}}{2 \Gamma((n-1) / 2)} \varepsilon^{n-2} \int_{\varepsilon}^{\infty} e^{-2 \pi\|x\| s} \frac{\left(s^{2}-\varepsilon^{2}\right)^{(n-3) / 2}}{\varepsilon^{n-3}} \frac{d s}{\varepsilon} \\
& \leqq \frac{\pi^{(n-3) / 2}}{2 \Gamma((n-1) / 2)} \int_{0}^{\infty} e^{-2 \pi\|x\| s} s^{n-3} d s \\
& =\frac{\pi^{(n-3) / 2} \Gamma(n-2)}{2 \Gamma((n-1) / 2)(2 \pi\|x\|)^{n-2}}
\end{aligned}
$$

nach Definition der Gammafunktion. Also gilt

$$
k_{\varepsilon}(x) \leqq \frac{\Gamma(n-2)}{(4 \pi)^{(n-1) / 2} \Gamma((n-1) / 2)\|x\|^{n-2}}=B \frac{1}{\|x\|^{n-2}} \quad(x \neq 0) .
$$

Weiters folgt aus der Integraldarstellung

$$
\begin{aligned}
k_{\varepsilon}(x) & =\frac{\pi^{(n-3) / 2}}{2 \Gamma((n-1) / 2)} \int_{0}^{\infty} e^{-2 \pi\|x\|(t+\varepsilon)}\left(t^{2}+2 t \varepsilon\right)^{(n-3) / 2} d t \\
& =\frac{\pi^{(n-3) / 2}}{2 \Gamma((n-1) / 2)} e^{-2 \pi\|x\| \varepsilon} \int_{0}^{\infty} e^{-2 \pi\|x\| t}\left(t^{2}+2 t \varepsilon\right)^{(n-3) / 2} d t .
\end{aligned}
$$

Nach dem Hilfssatz in 3.4, angewandt auf $n=1$, ist

$$
k_{1 / m}(x)=\frac{\pi^{(n-3) / 2}}{4 \Gamma((n-1) / 2)} e^{-2 \pi\|x\|(1 / m)} \int e^{-2 \pi\|x\||y|}\left(y^{2}+(2 / m)|y|\right)^{(n-3) / 2} d y,
$$

woraus nach dem Satz über majorisierte Konvergenz ([1], S. 122, (13.8.4)) folgt

$$
\lim _{m \rightarrow \infty} k_{1 / m}(x)=\frac{\pi^{(n-3) / 2}}{4 \Gamma((n-1) / 2)} \int e^{-2 \pi\|x\||y|}|y|^{n-3} d y .
$$

Rückverwandlung in ein uneigentliches Riemannintegral nach dem Hilfssatz liefert

$$
\begin{aligned}
\lim _{m \rightarrow \infty} k_{1 / m}(x) & =\frac{\pi^{(n-3) / 2}}{2 \Gamma((n-1) / 2)} \int_{0}^{\infty} e^{-2 \pi\|x\| t} t^{n-3} d t \\
& =\frac{\pi^{(n-3) / 2} \Gamma(n-2)}{2 \Gamma((n-1) / 2)(2 \pi\|x\|)^{n-2}}=\frac{B}{\|x\|^{n-2}} .
\end{aligned}
$$

Für $\psi \in \mathscr{S}$ wird nach dem Satz über majorisierte Konvergenz

$$
\lim _{m \rightarrow \infty}\left\langle\psi, k_{1 / m}\right\rangle=\lim _{m \rightarrow \infty} \int k_{1 / m} \psi d x=\int\left(\lim _{m \rightarrow \infty} k_{1 / m}\right) \psi d x,
$$

da wegen $(*)(x \neq 0)$

$$
k_{1 / m}(x)|\psi(x)| \leqq K\left(\sup _{x \in R^{n}}\left|\left(1+\|x\|^{4}\right) \psi(x)\right|\right) \frac{1}{\left(1+\|x\|^{4}\right)\|x\|^{n-2}}
$$


mit einer Konstanten $K$ gilt. Auch ist

Damit ist gezeigt, daß durch

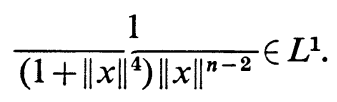

$$
E_{n}(\psi):=\int \frac{B}{\|x\|^{n-2}} \psi d x \quad \text { für } \quad \psi \in \mathscr{S}
$$

eine stetige Linearform auf $\mathscr{S}$ definiert ist, das heißt: $E_{n} \in \mathscr{S}^{\prime}$.

4.5. Aus der Gleichung

$$
\frac{4 \pi^{2}}{m^{2}}\left\langle\psi, k_{1 / m}\right\rangle-\left\langle\psi, \Delta_{n} k_{1 / m}\right\rangle=\langle\psi, \delta\rangle
$$

für $\psi \in \mathscr{S}$ folgt daher

sowie

$$
\lim _{m \rightarrow \infty} \frac{4 \pi^{2}}{m^{2}}\left\langle\psi, k_{1 / m}\right\rangle-\lim _{m \rightarrow \infty}\left\langle\Delta_{n} \psi, k_{1 / m}\right\rangle=\langle\psi, \delta\rangle,
$$

$$
\lim _{m \rightarrow \infty} \frac{4 \pi^{2}}{m^{2}} \lim _{m \rightarrow \infty}\left\langle\psi, k_{1 / m}\right\rangle-\lim _{m \rightarrow \infty}\left\langle\Delta_{n} \psi, k_{1 / m}\right\rangle=\langle\psi, \delta\rangle
$$

und

Daher ist

$$
-\int\left(\lim _{m \rightarrow \infty} k_{1 / m}\right) \Delta_{n} \psi d x=\langle\psi, \delta\rangle
$$

oder

$$
-\left\langle\Delta_{n} \psi, E_{n}\right\rangle=\langle\psi, \delta\rangle
$$$$
-\left\langle\psi, \Delta_{n} E_{n}\right\rangle=\langle\psi, \delta\rangle \text {. }
$$

Für $n \geqq 3$ ist $-E_{n}$ eine Fundamentallösung des Laplaceoperators in $\mathscr{S}^{\prime}$.

Bemerkung. Für die in (*) eingeführte Konstante $B$ findet man mittels

$$
\Gamma(n-2)=\Gamma(2(n-1 / 2))=\frac{2^{n-3}}{\sqrt{\pi}} \Gamma(n / 2-1) \Gamma((n-1) / 2)
$$

nach [2], S. 938, Formel 8.335, 1, den Wert

$$
B=\frac{1}{(n-2) \omega_{n}}
$$

4.6. In den Fällen $n=1$ und $n=2$ müssen wir etwas anders vorgehen, da die Grenzwerte

$$
\lim _{m \rightarrow \infty} \frac{m}{4 \pi} e^{-2 \pi|x| / m} \quad \text { und } \quad \lim _{m \rightarrow \infty} \frac{1}{2 \pi} K_{0}\left(\frac{2 \pi}{m}\|x\|\right) \quad(x \neq 0)
$$

nicht existieren. 
Im Fall $n=1$ ist

$$
\lim _{m \rightarrow \infty} \frac{m}{4 \pi}\left(e^{-2 \pi|x| / m}-1\right)=-\frac{1}{2}|x|
$$

$\mathrm{Da}$

ist, folgt für $\psi \in \mathscr{S}$

$$
\left|\frac{m}{4 \pi}\left(e^{-2 \pi|x| / m}-1\right)\right| \leqq \frac{1}{2}|x|
$$

$$
\left|\psi(x) \frac{m}{4 \pi}\left(e^{-2 \pi|x| / m}-1\right)\right| \leqq \frac{1}{2}|x||\psi(x)|
$$

Nach [15], S. 275, Theorem 25.5, und wegen $\mathscr{S} \subset L^{1}$ ist $x \psi \in L^{1}$.

Für $n=2$ gestaltet sich die Betrachtung schwieriger: Wegen der Reihendarstellung von $K_{0}$ nach [2], S. 961, Formel 8.447, 3, gilt

$$
K_{0}(2 \pi \varepsilon\|x\|)=-(\log \pi \varepsilon\|x\|) I_{0}(2 \pi \varepsilon\|x\|)+\sum_{j=0}^{\infty}(2 \pi \varepsilon\|x\|)^{2 j} \frac{\Psi(j+1)}{2^{2 j}(j !)^{2}}
$$

für $x \neq 0$, wobei $I_{0}$ die modifizierte Besselfunktion erster Art und $\Psi$ die Psifunktion sind. Es ist $I_{0}(0)=1$ und $\Psi(1)=-C$ mit der Eulerschen Konstanten $C$ ([2], S. 946, Formel $8.367,1)$. Also ist für $x \neq 0$

$$
\lim _{\varepsilon \downarrow 0}\left(K_{0}(2 \pi \varepsilon\|x\|)+\log \varepsilon\right)=-\log \pi-\log \|x\|-C
$$

oder

$$
\lim _{\varepsilon \downarrow 0}\left(K_{0}(2 \pi \varepsilon\|x\|)+\log \varepsilon+\log \pi+C\right)=-\log \|x\| .
$$

Als nächstes soll $k_{\varepsilon}(x)$ abgeschätzt werden: Aus der weiter oben angegebenen Integraldarstellung folgt

$$
\begin{aligned}
k_{\varepsilon}(x) & =\frac{1}{2 \pi} K_{0}(2 \pi \varepsilon\|x\|)=\frac{1}{2 \pi} \int_{1}^{\infty} e^{-2 \pi \varepsilon\|x\| t} \frac{d t}{\sqrt{t^{2}-1}} \\
& =\frac{1}{2 \pi} \int_{\varepsilon}^{\infty} e^{-2 \pi \tau\|x\|} \frac{d \tau}{\sqrt{\tau^{2}-\varepsilon^{2}}} \quad(x \neq 0) .
\end{aligned}
$$

Wir zerlegen dieses Integral in 3 Teile:

$$
k_{\varepsilon}(x)=\frac{1}{2 \pi}\left(\int_{\varepsilon}^{1} \frac{d \tau}{\sqrt{\tau^{2}-\varepsilon^{2}}}+\int_{\varepsilon}^{1} \frac{e^{-2 \pi\|x\| \tau}-1}{\sqrt{\tau^{2}-\varepsilon^{2}}} d \tau+\int_{1}^{\infty} \frac{e^{-2 \pi\|x\| \tau}}{\sqrt{\tau^{2}-\varepsilon^{2}}} d \tau\right)
$$

für $0<\varepsilon<1$. Der erste Summand wird

$$
\int_{\varepsilon}^{1} \frac{d \tau}{\sqrt{\tau^{2}-\varepsilon^{2}}}=\log \frac{1+\sqrt{1-\varepsilon^{2}}}{\varepsilon}
$$


Wegen

folgt

$$
\frac{1}{2 \pi}\left|e^{-2 \pi\|x\| \tau}-1\right| \leqq\|x\| \tau
$$

$$
\begin{gathered}
\left|k_{\varepsilon}(x)+\frac{1}{2 \pi}(\log \varepsilon+\log \pi+C)\right| \\
\leqq \frac{1}{2 \pi}\left(\log \left(1+\sqrt{1-\varepsilon^{2}}\right)+\log \pi+C+\|x\| \sqrt{1-\varepsilon^{2}}+\frac{e^{-2 \pi\|x\|}}{2 \pi\|x\| \sqrt{1-\varepsilon^{2}}}\right) .
\end{gathered}
$$

Speziell für $\varepsilon \leqq 1 / 2$ folgt

$$
\left|k_{\varepsilon}(x)+\frac{1}{2 \pi}(\log \varepsilon+\log \pi+C)\right| \leqq \frac{1}{2 \pi}\left(\log 2 \pi+C+\|x\|+\frac{1}{\pi \sqrt{3}\|x\|}\right) .
$$

Für $\psi \in \mathscr{S}$ ist

$$
(\log 2 \pi+C+\|x\|) \psi \in L^{1}
$$

wegen [15], S. 275, Theorem 25.5, und $\mathscr{S} \subset L^{1}$. Weiters ist nach dem Hilfssatz in $3.4 \psi /\|x\| \in L^{1}$.

Wir setzen nun

$$
\alpha_{m}:=\left\{\begin{array}{lll}
-\frac{m}{4 \pi} & \text { für } & n=1 \\
\frac{1}{2 \pi}\left(\log \frac{1}{m}+\log \pi+C\right) & \text { für } & n=2
\end{array}\right.
$$

und

$$
f(x):=\left\{\begin{array}{lll}
-\frac{1}{2}|x| & \text { für } & n=1 \\
-\frac{1}{2 \pi} \log \|x\| & \text { für } & n=2 .
\end{array}\right.
$$

Wegen der angegebenen Majorisierungen durch $L^{1}$-Funktionen, die von $m$ unabhängig sind, gilt für $\psi \in \mathscr{S}$

Weiters ist

$$
\lim _{m \rightarrow \infty} \int\left(k_{1 / m}(x)+\alpha_{m}\right) \psi d x=\int f \psi d x
$$

$$
\frac{4 \pi^{2}}{m^{2}}\left\langle\psi, k_{1 / m}\right\rangle-\left\langle\psi, \Delta_{n} k_{1 / m}\right\rangle=\langle\psi, \delta\rangle \quad \text { und } \quad \Delta_{n} \alpha_{m}=0
$$

Also wird

$$
\frac{4 \pi^{2}}{m^{2}}\left\langle\psi, k_{1 / m}\right\rangle+\frac{4 \pi^{2}}{m^{2}}\left\langle\psi, \alpha_{m}\right\rangle-\frac{4 \pi^{2}}{m^{2}}\left\langle\psi, \alpha_{m}\right\rangle-\left\langle\psi, \Delta_{n} k_{1 / m}\right\rangle-\left\langle\psi, \Delta_{n} \alpha_{m}\right\rangle=\langle\psi, \delta\rangle
$$

und

$$
\begin{gathered}
\frac{4 \pi^{2}}{m^{2}} \int \psi(x)\left(k_{1 / m}(x)+\alpha_{m}\right) d x-\frac{4 \pi^{2}}{m^{2}} \alpha_{m}\langle\psi, 1\rangle \\
-\int\left(\Delta_{n} \psi\right)(x)\left(k_{1 / m}(x)+\alpha_{m}\right) d x=\langle\psi, \delta\rangle .
\end{gathered}
$$


Folglich ist

und

$$
\left(\lim _{m \rightarrow \infty} \frac{4 \pi^{2}}{m^{2}}\right) \int \psi(x) f(x) d x-\lim _{m \rightarrow \infty}\left(\frac{4 \pi^{2}}{m^{2}} \alpha_{m}\right)\langle\psi, 1\rangle-\int\left(\Delta_{n} \psi\right) f d x=\langle\psi, \delta\rangle
$$

$$
\int \Delta_{n} \psi(-f) d x=\langle\psi, \delta\rangle
$$

Im Falle $n=1$ ist $-f$ im Unendlichen schwach wachsend, also erhalten wir

und

$$
\int \Delta_{n} \psi(-f) d x=\left\langle\Delta_{n} \psi,-f\right\rangle=\left\langle\psi, \Delta_{n}(-f)\right\rangle
$$

$$
\frac{d^{2}}{d x^{2}}\left(\frac{|x|}{2}\right)=\delta
$$

Für $n=1$ ist eine Fundamentallösung des Laplaceoperators in $\mathscr{S}^{\prime}$ durch $|x| / 2$ gegeben.

Weil

$$
\int_{0}^{\infty} \frac{t|\log t|}{1+t^{4}} d t=\frac{1}{4}\left(-\int_{0}^{1} \frac{\log t}{1+t^{2}} d t+\int_{1}^{\infty} \frac{\log t}{1+t^{2}} d t\right)=\frac{1}{2} G
$$

([2], S. 532, Formel 4.231, 12) mit der Catalanschen Konstanten $G$ ist, folgt für $n=2$ und $\psi \in \mathscr{S}$ nach dem Hilfssatz aus 3.4

$$
\int|\log \|x\| \psi| d x \leqq C \int \frac{|\log \|x\||}{1+\|x\|^{4}} d x=C \omega_{2} \int_{0}^{\infty} \frac{t|\log t|}{1+t^{4}} d t,
$$

und folglich ist $E_{2} \in \mathscr{S}^{\prime}$, wenn

$$
E_{2}(\psi):=\frac{1}{2 \pi} \int \log \|x\| \psi d x
$$

gesetzt wird. Es gilt somit

$$
\left\langle\psi, \Delta_{2} E_{2}\right\rangle=\left\langle\Delta_{2} \psi, E_{2}\right\rangle=\langle\psi, \delta\rangle
$$

Für $n=2$ ist $E_{2}$ eine Fundamentallösung des Laplaceoperators in $\mathscr{S}^{\prime}$.

\section{Die Existenz von Fundamentallösungen in $\mathscr{D}^{\prime}$}

Neben Problemen der tatsächlichen Konstruktion von Fundamentallösungen wenden wir uns jetzt der Frage der Existenz von Fundamentallösungen im allgemeinen zu. Wir werden zeigen, daß jeder nichttriviale Differentialoperator mit konstanten Koeffizienten eine Fundamentallösung in $\mathscr{D}^{\prime}$ besitzt. 
5.1. Zunächst ist klar, daß für $E \in \mathscr{D}^{\prime}$ die Aussagen

und

$$
P(\partial) E=\delta
$$

$$
\langle\varphi, P(\partial) E\rangle=\langle\varphi, \delta\rangle=\varphi(0) \text { für alle } \varphi \in \mathscr{D}
$$

und - nach Definition der Transponierten -

$$
\left\langle P^{\curlyvee}(\partial) \varphi, E\right\rangle=\langle P(-\partial) \varphi, E\rangle=\varphi(0) \text { für alle } \varphi \in \mathscr{D}
$$

äquivalent sind. Hat $P(\partial)$ eine Fundamentallösung $E$, so gilt also

$$
\langle\psi, E\rangle=\varphi(0)
$$

für alle $\psi \in \mathscr{D}$ der Form $\psi:=P(-\partial) \varphi$ mit $\varphi \in \mathscr{D}$.

Daraus kann man leicht vermuten:

Satz 2. Falls die durch

$$
L: P(-\partial) \mathscr{D} \rightarrow C, \psi \mapsto \varphi(0)
$$

für $\psi=P(-\partial) \varphi$ und $\varphi \in \mathscr{D}$ auf dem Untervektorraum $P(-\partial) \mathscr{D}$ (versehen mit der von $\mathscr{D}$ induzierten Topologie) definierte Linearform stetig ist, existiert eine Fundamentallösung des Differentialoperators $P(\partial)$.

Beweis. (1) $L$ ist wohldefiniert: Gilt für $\varphi_{1}, \varphi_{2} \in \mathscr{D}$

$$
\psi=P(-\partial) \varphi_{1}=P(-\partial) \varphi_{2},
$$

so erhält man durch Fouriertransformation

$$
P(-2 \pi i \xi) \mathscr{F}\left(\varphi_{1}-\varphi_{2}\right)=0 .
$$

Ist $N$ die Nullstellenmenge der Funktion $\xi \mapsto P(-2 \pi i \xi)$, so gilt

$$
\mathscr{F}\left(\varphi_{1}-\varphi_{2}\right)=0 \text { in } \complement N \text {. }
$$

Da $\complement N$ dicht in $\boldsymbol{R}^{n}$ (vgl. [8], S. 11, 2.2.) und $\xi \mapsto \mathscr{F}\left(\varphi_{1}-\varphi_{2}\right)(\xi)$ gleichmäß1g stetig ist, wird

$$
\mathscr{F}\left(\varphi_{1}-\varphi_{2}\right)=0,
$$

woraus wegen der Isomorphieeigenschaft der Fouriertransformation

folgt.

$$
\varphi_{1}=\varphi_{2} \quad \text { und speziell } \quad \varphi_{1}(0)=\varphi_{2}(0)
$$

(2) Ist $L$ stetig auf $P(\partial) \mathscr{D}$, so hat $L$ nach dem Satz von Hahn-Banach eine stetige Fortsetzung $E$ auf ganz $\mathscr{D}$. Wegen der Fortsetzungseigenschaft ist für $\varphi \in \mathscr{D}$

$$
\langle P(-\partial) \varphi, E\rangle=\langle P(-\partial) \varphi, L\rangle=\varphi(0),
$$

womit $E$ eine Fundamentallösung ist. 
Bemerkung. Der Satz zeigt, daß sich die Frage nach der Existenz einer Fundamentallösung des linearen partiellen Differentialoperators $P(\partial)$ auf den Nachweis der Stetigkeit des linearen Funktionals $L$ reduziert.

5.2. In den nächsten Sätzen soll nun die Stetigkeit von $L$ gezeigt werden.

Satz 3. Für $\psi \in \mathscr{S}$ und $a \in \boldsymbol{R}^{n}$ ist

$$
|\psi(a)| \leqq C_{n}\left(\sum_{|j| \leqq[n / 2]+1} \int\left|\partial^{j} \psi\right|^{2} d x\right)^{1 / 2}=C_{n}\left(\sum_{|j| \leqq[n / 2]+1}\left\|\partial^{j} \psi\right\|_{2}^{2}\right)^{1 / 2},
$$

wo $C_{n}$ eine nur von der Dimension des Raumes $\boldsymbol{R}^{n}$ abhängige Konstante ist.

Beweis. Nach [15], S. 268, Theorem 25.1, wird

$$
\psi(x)=\int e^{2 \pi i x \xi}(\mathscr{F} \psi)(\xi) d \xi
$$

für $x \in \boldsymbol{R}^{n}$. Für $a \in \boldsymbol{R}^{n}$ folgt daraus

$$
|\psi(a)| \leqq \int|\mathscr{F} \psi| d \xi .
$$

Für $\operatorname{Re} p>0$ und $\operatorname{Re} q>0$ ist

$$
B(p, q)=\int_{0}^{1} t^{p-1}(1-t)^{q-1} d t
$$

das Eulersche Integral erster Gattung. Wir setzen $t=r^{2} /\left(1+r^{2}\right)$. Dann wird

$$
B(p, q)=2 \int_{0}^{\infty} \frac{r^{2 p-1} d r}{\left(1+r^{2}\right)^{p+q}}
$$

Daraus folgt

$$
\int_{0}^{\infty} r^{n-1}\left(1+r^{2}\right)^{-s} d r=\frac{1}{2} B\left(\frac{n}{2}, s-\frac{n}{2}\right) \quad \text { für } \quad s \in \boldsymbol{R} \quad \text { mit } \quad s-n / 2>0 .
$$

Nach dem Hilfssatz in 3.4 ist daher

und

$$
\left(1+\|\xi\|^{2}\right)^{-s} \in L^{1} \quad \text { für } \quad s>n / 2
$$

$$
\int\left(1+\|\xi\|^{2}\right)^{-s} d \xi=\frac{\omega_{n}}{2} B\left(\frac{n}{2}, s-\frac{n}{2}\right)=\pi^{n / 2} \frac{\Gamma(s-n / 2)}{\Gamma(s)}
$$

([2], S. 950, Formel 8.384, 1). Also ist

$$
\left(1+\|\xi\|^{2}\right)^{-s / 2} \in L^{2},
$$

wenn $s>n / 2$ ist. Da wegen $\mathscr{S} \subset L^{2}$

gilt, folgt aus

$$
\mathscr{F} \psi \in L^{2} \quad \text { und } \quad\left(1+\|\xi\|^{2}\right)^{s / 2} \mathscr{F} \psi \in L^{2}
$$

$$
|\psi(a)| \leqq \int|\mathscr{F} \psi| d \xi=\int\left(1+\|\xi\|^{2}\right)^{-s / 2}\left(1+\|\xi\|^{2}\right)^{s / 2}|(\mathscr{F} \psi)(\xi)| d \xi
$$


nach der Hölderschen Ungleichung

$$
\begin{aligned}
& |\psi(a)| \leqq\left\|\left(1+\|\xi\|^{2}\right)^{-s / 2}\right\|_{2}\left\|\left(1+\|\xi\|^{2}\right)^{s / 2} \mathscr{F} \psi\right\|_{2} \\
& =\pi^{n / 4} \sqrt{\frac{\Gamma(s-n / 2)}{\Gamma(s)}}\left(\int\left(1+\|\xi\|^{2}\right)^{s}|\mathscr{F} \psi|^{2} d \xi\right)^{1 / 2}
\end{aligned}
$$

Elementare Formeln ergeben mit $s \in N$

$$
\left(1+\|\xi\|^{2}\right)^{s}=\sum_{v=0}^{s}\left(\begin{array}{l}
s \\
v
\end{array}\right) \sum_{|j|=v} \frac{v !}{j !} \xi^{2 j} \leqq\left(\max _{0 \leqq v \leqq s}\left(\begin{array}{l}
s \\
v
\end{array}\right)\right) s ! \sum_{|j| \leqq s} \xi^{2 j} .
$$

Eine mögliche Wahl für $s$ ist daher $s=[n / 2]+1$. Sei

Dann gilt,

$$
C_{n}:=\pi^{n / 4}\left(\frac{\Gamma([n / 2]+1-n / 2)}{\Gamma([n / 2]+1)} \max _{0 \leqq v \leqq[n / 2]+1}\left(\begin{array}{c}
{[n / 2]+1} \\
v
\end{array}\right)([n / 2]+1) !\right)^{1 / 2} .
$$

$$
|\psi(a)| \leqq C_{n}\left(\sum_{|j| \leqq[n / 2]+1} \int \xi^{2 j}|\mathscr{F} \psi|^{2} d \xi\right)^{1 / 2}=C_{n}\left(\sum_{|j| \leqq[n / 2]+1}\left\|\xi^{j} \mathscr{F} \psi\right\|_{2}^{2}\right)^{1 / 2} .
$$

Nach [15], S. 277, Theorem 25.7, ergibt sich

$$
\xi^{j} \mathscr{F} \psi=\frac{1}{(2 \pi i)^{|j|}} \mathscr{F}\left(\partial^{j} \psi\right)
$$

nach der Parsevalschen Formel ist

also auch

$$
\left\|\mathscr{F}\left(\partial^{j} \psi\right)\right\|_{2}^{2}=\left\|\partial^{j} \psi\right\|_{2}^{2},
$$

Zusammenfassend erhalten wir

$$
\left\|\xi^{j} \mathscr{F} \psi\right\|_{2}^{2} \leqq\left\|\partial^{j} \psi\right\|_{2}^{2} .
$$

$$
|\psi(a)| \leqq C_{n}\left(\sum_{|j| \leqq[n / 2]+1}\left\|\partial^{j} \psi\right\|_{2}^{2}\right)^{1 / 2} .
$$

Bemerkung. Die Aussage des letzten Satzes kann auch folgendermaßen formuliert werden:

Wird $\mathscr{S}$ mit der von der Norm

$$
\|\cdot\|_{[n / 2]+1}: \mathscr{S} \mapsto[0, \infty), \quad \psi \mapsto\left(\sum_{|j| \leqq[n / 2]+1}\left\|\partial^{j} \psi\right\|_{2}^{2}\right)^{1 / 2}
$$

erzeugten Topologie (die gröber als die Topologie von $\mathscr{S}$ ist) versehen, so ist $\delta_{a}$ bereits in dieser Topologie stetig. Die Vervollständigung von $\mathscr{S}$ unter dieser Topologie ist der Sobolevraum

$$
H^{[n / 2]+1}=W^{[n / 2]+1,2} .
$$

Also läßt sich $\delta_{a}$ stetig auf $H^{[n / 2]+1}$ fortsetzen. Wird die Fortsetzung von $\delta_{a}$ wieder mit $\delta_{a}$ bezeichnet, so gilt also

$$
\delta_{a} \in\left(H^{[n / 2]+1}\right)^{\prime}=H^{-[n / 2]-1} .
$$


Bezieht man Sobolevräume beliebiger Ordnung in die Betrachtung ein, so gilt

$$
\delta_{a} \in H^{-(n / 2)-\varepsilon} \text { mit } \varepsilon>0 .
$$

5.3. Den entscheidenden Schritt bewältigen wir in

Satz 4. Sei

$$
P(\partial) \varphi:=\sum_{|j| \leqq m} a_{j} \partial^{j} \varphi, \quad a_{j} \in C, \quad \varphi \in \mathscr{D}
$$

ein linearer partieller Differentialoperator der Ordnung $m \in N_{0}$. Mit jedem $\varepsilon>0$ gilt für alle $p \in N_{0}^{n}$ und alle $\varphi \in \mathscr{D}$ :

$$
\left\|P^{(p)}(\partial) \varphi\right\|_{2} \leqq\left(\frac{2 m}{\varepsilon}\right)^{|p|}\left\|e^{\varepsilon\|x\|} P(\partial) \varphi\right\|_{2} .
$$

Dabei ist $P^{(p)}=\partial^{p} P$ für $p \in N_{0}^{n}$.

Beweis. Es genügt, die Ungleichung für $p=(1,0, \ldots, 0) \mathrm{zu}$ beweisen und dann den allgemeinen Fall durch Induktion herzuleiten.

Die Modifikation des Hörmanderschen Beweises durch Treves beruht auf folgenden Beobachtungen:

(a) $P(\partial) \varphi=\varphi * P(\partial) \delta$ für $\varphi \in \mathscr{D}$,

(b) $P(\partial) \delta$ ist eine Distribution mit kompaktem Träger,

(c) $x_{1} P(\partial) \delta=P^{(1,0, \ldots, 0)}(\partial) \delta$, also nach (a)

$$
P^{(1,0, \ldots, 0)}(\partial) \varphi=\varphi *\left(x_{1} P(\partial) \delta\right) .
$$

Wir beweisen (c): Es ist

Mit $\psi \in \mathscr{D}$ ist

$$
P^{(1,0, \ldots, 0)}(\xi)=\sum_{1 \leqq|j| \leqq m} j_{1} a_{j} \xi_{1}^{j_{1}-1} \xi_{2}^{j_{2}} \ldots \xi_{n}^{j_{n}}
$$

$$
\begin{aligned}
\left\langle\psi, x_{1} P(\partial) \delta\right\rangle & =\left\langle x_{1} \psi, P(\partial) \delta\right\rangle=\left\langle P^{\smile}(\partial)\left(x_{1} \psi\right), \delta\right\rangle \\
& =\left\langle\sum_{|j| \leqq m}(-1)^{|j|} a_{j} \partial^{j}\left(x_{1} \psi\right), \delta\right\rangle .
\end{aligned}
$$

Nach der Leibnizschen Formel ist

also haben wir

$$
\begin{aligned}
\partial^{j}\left(x_{1} \psi\right) & =\sum_{l \leqq j}\left(\begin{array}{l}
j \\
l
\end{array}\right) \partial^{l} x_{1} \partial^{j-l} \psi \\
& =x_{1} \partial^{j} \psi+\left(\begin{array}{c}
j \\
(1,0, \ldots, 0)
\end{array}\right) \partial^{j_{1}-1+j_{2}+\ldots+j_{n}} \psi
\end{aligned}
$$

wegen

$$
\left\langle\psi, x_{1} P(\partial) \delta\right\rangle=\left\langle\sum_{|j| \leqq m} a_{j}(-1)^{|j|} j_{1} \partial^{j_{1}-1+j_{2}+\ldots+j_{n}} \psi, \delta\right\rangle,
$$

$$
\begin{aligned}
\left\langle x_{1} \partial^{j} \psi, \delta\right\rangle= & 0 \quad \text { und }\left(\begin{array}{c}
j \\
(1,0, \ldots, 0)
\end{array}\right)=j_{1} ; \quad \text { somit ergibt sich } \\
& \left\langle\psi, x_{1} P(\partial) \delta\right\rangle=\left\langle\psi, P^{(1,0, \ldots, 0)}(\partial) \delta\right\rangle .
\end{aligned}
$$


Mittels der Aussagen (a)-(c) beweisen wir die Ungleichung

$$
\left\|\varphi *\left(x_{1} T\right)\right\|_{2} \leqq C\left\|e^{\varepsilon\|x\|}(\varphi * T)\right\|_{2}
$$

für eine Distribution $T$ mit kompaktem Träger. In der Tat werden wir auf den nächsten Seiten

$$
\left\|\varphi *\left(x_{1} T\right)\right\|_{2}^{2} \leqq\left\|\mathrm{e}^{\varepsilon\|x\|}(\varphi * T)\right\|_{2}\left(\frac{2}{\varepsilon}\left\|\varphi *\left(x_{1} T\right)\right\|_{2}+\left\|\varphi *\left(x_{1}^{2} T\right)\right\|_{2}\right)
$$

beweisen. Setzt man für $T$ wieder $P(\partial) \delta$ ein und führt noch einen Induktionsbeweis über den Grad $m$ des Operators $P(\partial)$, so ergibt sich die gesuchte Ungleichung.

Zum Beweis von (*) benötigen wir 3 Hilfssätze:

Lemma 1. Für $\varphi \in \mathscr{D}$ und $T \in \mathscr{D}^{\prime}$ gilt

$$
x_{1}(\varphi * T)=\varphi *\left(x_{1} T\right)+\left(x_{1} \varphi\right) * T .
$$

Beweis. Nach Definition ist

und daher

$$
(\varphi * T)(x)=\langle(y \mapsto \varphi(x-y)), T\rangle
$$

$$
\begin{aligned}
\left(x_{1}(\varphi * T)\right)(x) & =x_{1}\langle(y \mapsto \varphi(x-y)), T\rangle=\left\langle\left(y \mapsto x_{1} \varphi(x-y)\right), T\right\rangle \\
& =\left\langle\left(y \mapsto\left(x_{1}-y_{1}\right) \varphi(x-y)\right), T\right\rangle+\left\langle\left(y \mapsto y_{1} \varphi(x-y)\right), T\right\rangle \\
& =\left(\left(x_{1} \varphi\right) * T\right)(x)+\left(\varphi *\left(x_{1} T\right)\right)(x) .
\end{aligned}
$$

Lemma 2. Sei

$$
(v, w)_{2}:=\int v \bar{w} d x \quad \text { für } \quad v, w \in L^{2}
$$

und weiters $\varphi \in \mathscr{D}, T \in \mathscr{D}^{\prime}, \psi \in \mathscr{D}, S \in \mathscr{E}^{\prime}$. Dann ist

$$
(\varphi * S, \psi * T)_{2}=\left(\psi * S^{\sim}, \varphi * T^{\sim}\right)_{2},
$$

wobei $T^{\sim}$ durch $T^{\sim}(\varrho):=T\left(\overline{\varrho^{\smile}}\right)$ für $\varrho \in \mathscr{D}$ definiert ist $\left(\varrho^{\smile}(x)=\varrho(-x)\right)$. Insbesondere wird

$$
\|\varphi * S\|_{2}=\left\|\varphi * S^{\sim}\right\|_{2}
$$

Beweis. (1) Ist $g$ stetig mit kompaktem Träger und $f$ stetig, so wird

$$
(g, f)_{2}=\int g(x) \overline{f(x)} d x=\int g^{\smile}(-x) \overline{f(x)} d x=\left(g^{\llcorner} * \bar{f}\right)(0) .
$$

Diese Gleichung kann auch in der Form

geschrieben werden.

$$
\left(g^{\smile}, \bar{f}\right)_{2}=(g * f)(0)=(f * g)(0)
$$

(2) Da supp $S$ kompakt ist, folgt $\varphi * S \in \mathscr{D}$ nach [15], S. 288, Satz 27.1.

(3) Also wird

$$
\left.(\varphi * S, \psi * T)_{2}=\left((\varphi * S)^{\swarrow} * \overline{(\psi * T}\right)\right)(0) .
$$


(4) Es ist

$$
\begin{aligned}
(\varphi * S)^{\smile}(x) & =(\varphi * S)(-x)=\langle(y \mapsto \varphi(-x-y)), S\rangle \\
& =\left\langle y \mapsto \varphi^{\smile}(x-y), S^{\smile}\right\rangle=\left(\varphi^{\smile} * S^{\smile}\right)(x) .
\end{aligned}
$$

(5) Die Distributionen $\operatorname{Re} T$ und $\operatorname{Im} T$ seien definiert durch

Dann ist

$$
\begin{aligned}
& (\operatorname{Re} T)(\varrho):=\operatorname{Re}(T(\varrho)) \\
& (\operatorname{Im} T)(\varrho):=\operatorname{Im}(T(\varrho))
\end{aligned} \text { für reellwertige } \varrho \in \mathscr{D} .
$$

$$
\langle\sigma, \operatorname{Re} T\rangle=\langle\operatorname{Re} \sigma, \operatorname{Re} T\rangle+i\langle\operatorname{Im} \sigma, \operatorname{Re} T\rangle
$$$$
=\operatorname{Re}\langle\operatorname{Re} \sigma, T\rangle+i \operatorname{Re}\langle\operatorname{Im} \sigma, T\rangle \quad \text { für } \quad \sigma \in \mathscr{D} .
$$

Ebenso wird

$$
\langle\sigma, \operatorname{Im} T\rangle=\operatorname{Im}\langle\operatorname{Re} \sigma, T\rangle+i \operatorname{Im}\langle\operatorname{Im} \sigma, T\rangle,
$$

also

$$
\begin{gathered}
\langle\sigma, \operatorname{Re} T-i \operatorname{Im} T\rangle=\langle\overline{\operatorname{Re} \sigma, T}\rangle+i \overline{\langle\operatorname{Im} \sigma, T}\rangle \\
=\langle\overline{\operatorname{Re} \sigma, T\rangle-i\langle\operatorname{Im} \sigma, T}\rangle=\langle\overline{\bar{\sigma}, T}\rangle .
\end{gathered}
$$

Definiert man $\bar{T}:=\operatorname{Re} T-i \operatorname{Im} T$, so ergibt sich

$$
\langle\sigma, \bar{T}\rangle=\langle\overline{\bar{\sigma}, T}\rangle \quad \text { oder } \quad\langle\bar{\sigma}, \bar{T}\rangle=\langle\overline{\sigma, T}\rangle .
$$

(6) Also erhalten wir

und weiters

$$
(\varphi * S, \psi * T)_{2}=\left(\left(\varphi^{\smile} * S^{\smile}\right) *(\bar{\psi} * \bar{T})\right)(0)
$$

$$
\begin{aligned}
& \left(\varphi^{\smile} * S^{\smile}\right) *(\bar{\psi} * \bar{T})=(\bar{\psi} * \bar{T}) *\left(\varphi^{\smile} * S^{\smile}\right)=\left((\bar{\psi} * \bar{T}) * \varphi^{\smile}\right) * S^{\smile} \\
& =\left(\varphi^{\smile} *(\bar{\psi} * \bar{T})\right) * S^{\smile}=\left(\left(\varphi^{\smile} * \bar{\psi}\right) * \bar{T}\right) * S^{\smile}=\left(\left(\bar{\psi} * \varphi^{\smile}\right) * \bar{T}\right) * S \\
& =\left(\bar{\psi} *\left(\varphi^{\curlyvee} * \bar{T}\right) * S^{\smile}\right)=\left(\left(\varphi^{\curlyvee} * T^{\smile}\right) * \bar{\psi}\right) * S^{\smile}=\left(\varphi^{\smile} * \bar{T}\right) *\left(\bar{\psi} * S^{\smile}\right) \text {. }
\end{aligned}
$$

Daher folgt nach (1), (4) und (5)

$$
\begin{aligned}
(\varphi * S, \psi * T)_{2} & =\left(\left(\varphi^{\smile} * \bar{T}\right) *\left(\bar{\psi} * S^{\smile}\right)\right)(0)=\left(\left(\bar{\psi} * S^{\smile}\right)^{\smile}\right),\left(\overline{\varphi^{\smile} * \bar{T}}\right)_{2} \\
& =\left(\overline{\psi^{\smile}} * S, \overline{\varphi^{\smile}} * T\right)_{2}=\left(\psi * S^{\sim}, \varphi * T^{\sim}\right)_{2} .
\end{aligned}
$$

Lemma 3. Für $\varphi \in \mathscr{D}$ und $S \in \mathscr{E}^{\prime}$ ist $\varphi *\left(x_{1} S\right) \in L^{2}$.

Beweis. Nach Lemma 1 ist

$$
\left(x_{1}(\varphi * S), \varphi *\left(x_{1} S\right)\right)_{2}=\left(\varphi *\left(x_{1} S\right), \varphi *\left(x_{1} S\right)\right)_{2}+\left(\left(x_{1} \varphi\right) * S, \varphi *\left(x_{1} S\right)\right)_{2} .
$$

Somit erhalten wir

$$
\begin{aligned}
\left\|\varphi *\left(x_{1} S\right)\right\|_{2}^{2} & =\left(\varphi *\left(x_{1} S\right), \varphi *\left(x_{1} S\right)\right)_{2} \\
& =\left(x_{1}(\varphi * S), \varphi *\left(x_{1} S\right)\right)_{2}-\left(\left(x_{1} \varphi\right) * S, \varphi *\left(x_{1} S\right)\right)_{2} .
\end{aligned}
$$

Aus der Existenz der beiden rechtsstehenden Integrale folgt

$$
\varphi *\left(x_{1} S\right) \in L^{2} \text {. }
$$


Nach diesen Vorbereitungen beweisen wir nun den Satz in mehreren Schritten; sei dazu $\varphi \in \mathscr{D}$ und $T \in \mathscr{E}^{\prime}$ :

(1) Nach dem Beweis von Lemma 3 wird mittels Lemma 2 und 1

$$
\begin{aligned}
& \left(x_{1}(\varphi * T), \varphi *\left(x_{1} T\right)\right)_{2}=\left\|\varphi *\left(x_{1} T\right)\right\|_{2}^{2}+\left(\left(x_{1} \varphi\right) * T, \varphi *\left(x_{1} T\right)\right)_{2} \\
= & \left\|\varphi *\left(x_{1} T\right)\right\|_{2}^{2}+\left(\varphi * T^{\sim},\left(x_{1} \varphi\right) *\left(x_{1} T\right)^{\sim}\right)_{2} \\
= & \left\|\varphi *\left(x_{1} T\right)\right\|_{2}^{2}+\left(\varphi * T^{\sim}, x_{1}\left(\varphi *\left(x_{1} T\right)^{\sim}\right)\right)_{2}-\left(\varphi * T^{\sim}, \varphi *\left(x_{1}\left(x_{1} T\right)^{\sim}\right)\right)_{2} .
\end{aligned}
$$

(2) Wegen $\left|x_{1}\right| \leqq\|x\|$ und $\varepsilon\|x\| \leqq e^{\varepsilon\|x\|}$ ist mit $\varepsilon>0\left|x_{1}\right| \leqq(1 / \varepsilon) e^{\varepsilon\|x\|}$. Also ist $\varepsilon\left|x_{1}\right| e^{-\varepsilon\|x\|} \leqq 1$ oder $\left\|\varepsilon x_{1} e^{-\varepsilon\|x\|}\right\|_{\infty} \leqq 1$.

(3) Wir haben

$$
\left(\varphi * T^{\sim}, x_{1}\left(\varphi *\left(x_{1} T\right)^{\sim}\right)\right)_{2}=\left((1 / \varepsilon) e^{\varepsilon\|x\|}\left(\varphi * T^{\sim}\right), \varepsilon x_{1} e^{-\varepsilon\|x\|}\left(\varphi *\left(x_{1} T\right)^{\sim}\right)\right)_{2} .
$$

Nach Lemma 3 ist $\varphi *\left(x_{1} T\right)^{\sim} \in L^{2}$, also auch $x_{1} e^{-\varepsilon\|x\|}\left(\varphi *\left(x_{1} T\right)^{\sim}\right) \in L^{2}$ und natürlich wegen der Kompaktheit des Trägers von $\varphi * T^{\sim}$ noch $(1 / \varepsilon) e^{\varepsilon\|x\|}\left(\varphi * T^{\sim}\right) \in L^{2}$. Somit ergibt sich

$$
\begin{aligned}
\left|\left(\varphi * T^{\sim}, x_{1}\left(\varphi *\left(x_{1} T\right)^{\sim}\right)\right)_{2}\right| & \leqq\left\|(1 / \varepsilon) e^{\varepsilon\|x\|}\left(\varphi * T^{\sim}\right)\right\|_{2}\left\|\varepsilon x_{1} e^{-\varepsilon\|x\|}\right\|_{\infty}\left\|\varphi *\left(x_{1} T\right)^{\sim}\right\|_{2} \\
& \leqq(1 / \varepsilon)\left\|e^{\varepsilon\|x\|}\left(\varphi * T^{\sim}\right)\right\|_{2}\left\|\varphi *\left(x_{1} T\right)^{\sim}\right\|_{2} .
\end{aligned}
$$

(4) Wir schätzen $a b$ :

$$
\begin{aligned}
& \left|\left(x_{1}(\varphi * T), \varphi *\left(x_{1} T\right)\right)_{2}\right| \leqq\left\|x_{1}(\varphi * T)\right\|_{2}\left\|\varphi *\left(x_{1} T\right)\right\|_{2} \\
& \quad=\left\|x_{1} \varepsilon e^{-\varepsilon\|x\|}(1 / \varepsilon) e^{\varepsilon\|x\|}(\varphi * T)\right\|_{2}\left\|\varphi *\left(x_{1} T\right)\right\|_{2} \\
& \leqq(1 / \varepsilon)\left\|e^{\varepsilon\|x\|}(\varphi * T)\right\|_{2}\left\|\varphi *\left(x_{1} T\right)\right\|_{2} .
\end{aligned}
$$

(5) Es ist $\varphi *\left(x_{1}\left(x_{1} T\right)^{\sim}\right)=-\varphi * x_{1}^{2}\left(T^{\sim}\right)$ und nach Lemma 1

$$
\begin{aligned}
\varphi * x_{1}^{2} T^{\sim} & =x_{1}\left(\varphi *\left(x_{1} T^{\sim}\right)\right)-\left(x_{1} \varphi\right) *\left(x_{1} T^{\sim}\right) \\
& =x_{1}^{2}\left(\varphi * T^{\sim}\right)-x_{1}\left(\left(x_{1} \varphi\right) * T^{\sim}\right)-\left(x_{1} \varphi\right) * x_{1} T^{\sim} .
\end{aligned}
$$

Die drei Funktionen der rechten Seite gehören $\mathrm{zu} L^{2}$; die dritte nach Lemma 3 und die beiden ersten, da die Träger kompakt sind. Also folgt $\varphi * x_{1}^{2} T^{\sim} \in L^{2}$. Es wird

$$
\left|\left(\varphi * T^{\sim}, \varphi *\left(x_{1}\left(x_{1} T\right)^{\sim}\right)\right)\right|_{2} \leqq\left\|\varphi * T^{\sim}\right\|_{2}\left\|\varphi *\left(x_{1}^{2} T^{\sim}\right)\right\|_{2}
$$

(6) Es ist

$$
\leqq\left\|e^{\varepsilon\|x\|}\left(\varphi * T^{\sim}\right)\right\|_{2}\left\|\varphi *\left(x_{1}^{2} T^{\sim}\right)\right\|_{2} .
$$

da mit

$$
\left\|e^{\varepsilon\|x\|}\left(\varphi * T^{\sim}\right)\right\|_{2}=\left\|e^{\varepsilon\|x\|}(\varphi * T)\right\|_{2},
$$

nach Lemma 2 gilt

$$
A:=\operatorname{supp}\left(\left(\varphi * T^{\sim}\right)-(\varphi * T)\right)
$$

$$
\left\|e^{\varepsilon\|x\|}\left(\varphi * T^{\sim}-\varphi * T\right)\right\|_{2}^{2} \leqq \sup _{x \in A} e^{2 \varepsilon\|x\|}\left\|\varphi * T^{\sim}-\varphi * T\right\|_{2}^{2}=0 .
$$


(7) Gemäß Lemma 1 und 2 erhalten wir

$$
\begin{aligned}
\left\|\varphi *\left(x_{1} T^{\sim}\right)-\varphi *\left(x_{1} T\right)\right\|_{2}^{2} & =\left\|\varphi * x_{1}\left(T^{\sim}-T\right)\right\|_{2}^{2} \\
& =\left\|x_{1}\left(\varphi *\left(T^{\sim}-T\right)\right)-\left(x_{1} \varphi\right) *\left(T^{\sim}-T\right)\right\|_{2}^{2} \\
& \leqq 2\left(\sup _{x \in B}\left|x_{1}\right|\left\|\varphi *\left(T^{\sim}-T\right)\right\|_{2}^{2}+\left\|\left(x_{1} \varphi\right) *\left(T^{\sim}-T\right)\right\|_{2}^{2}\right)=0
\end{aligned}
$$

mit $B:=\sup \left(\varphi *\left(T^{\sim}-T\right)\right)$. Also ist

$$
\left\|\varphi *\left(x_{1} T^{\sim}\right)\right\|_{2}=\left\|\varphi *\left(x_{1} T\right)\right\|_{2} \quad \text { und } \quad\left\|\varphi *\left(x_{1} T\right)^{\sim}\right\|_{2}=\left\|\varphi *\left(x_{1} T\right)\right\|_{2} .
$$

(8) Ebenso wird nach Lemma 1

$$
\begin{gathered}
\left\|\varphi *\left(x_{1}^{2} T^{\sim}\right)-\varphi *\left(x_{1}^{2} T\right)\right\|_{2}^{2}=\left\|\varphi *\left(x_{1}^{2}\left(T^{\sim}-T\right)\right)\right\|_{2}^{2} \\
=\left\|x_{1}\left(\varphi *\left(x_{1}\left(T^{\sim}-T\right)\right)\right)-\left(x_{1} \varphi\right) *\left(x_{1}\left(T^{\sim}-T\right)\right)\right\|_{2}^{2} \\
=\left\|x_{1}\left(x_{1}\left(\varphi *\left(T^{\sim}-T\right)\right)-\left(x_{1} \varphi\right) *\left(T^{\sim}-T\right)\right)-\left(x_{1} \varphi\right) *\left(x_{1}\left(T^{\sim}-T\right)\right)\right\|_{2}^{2} \\
\leqq 2\left(\sup _{x \in B} x_{1}^{2}\left\|\varphi *\left(T^{\sim}-T\right)\right\|_{2}^{2}+\left\|\left(x_{1} \varphi\right) *\left(T^{\sim}-T\right)\right\|_{2}^{2}+\left\|\left(x_{1} \varphi\right) *\left(x_{1}\left(T^{\sim}-T\right)\right)\right\|_{2}^{2}\right),
\end{gathered}
$$

wobei die ersten 2 Ausdrücke nach Lemma 2 verschwinden und der dritte nach (7).

(9) Aus (1) folgt nach der Dreiecksungleichung

$$
\begin{aligned}
\left\|\varphi *\left(x_{1} T\right)\right\|_{2}^{2} & \leqq(1 / \varepsilon)\left\|e^{\varepsilon\|x\|}(\varphi * T)\right\|_{2}\left\|\varphi *\left(x_{1} T\right)\right\|_{2} \\
& +\left\|e^{\varepsilon\|x\|}\left(\varphi * T^{\sim}\right)\right\|_{2}\left\|\varphi *\left(x_{1}^{2} T^{\sim}\right)\right\|_{2}+(1 / \varepsilon)\left\|e^{\varepsilon\|x\|}\left(\varphi * T^{\sim}\right)\right\|_{2}\left\|\varphi *\left(x_{1} T^{\sim}\right)\right\|_{2}
\end{aligned}
$$

unter Verwendung von (4), (5) und (3).

(10) Daraus folgt mittels (6), (7) und (8)

$$
\left\|\varphi *\left(x_{1} T\right)\right\|_{2}^{2} \leqq\left\|e^{\varepsilon\|x\|}(\varphi * T)\right\|_{2}\left((2 / \varepsilon)\left\|\varphi *\left(x_{1} T\right)\right\|_{2}+\left\|\varphi *\left(x_{1}^{2} T\right)\right\|_{2}\right),
$$

womit die Ungleichung $(*)$ gezeigt ist.

(11) Sei nun $T=P(\partial) \delta$. Wir beweisen den Satz durch Induktion über den Grad $m$ des Differentialoperators $P(\partial)$. Sei also $m=1$, das heißt

Dann ist

$$
T=\sum_{|j| \leqq 1} a_{j} \partial^{j} \delta
$$

und

$$
\varphi * T=\sum_{|j| \leqq 1} a_{j}\left(\varphi * \partial^{j} \delta\right)=P(\partial) \varphi
$$

$$
x_{1} T=\sum_{|j|=1} a_{j} x_{1} \partial^{j} \delta=-a_{(1,0, \ldots, 0)} \delta,
$$

also nach c) am Anfang des Beweises

Weiters ist

$$
P^{(1,0, \ldots, 0)}(\partial) \varphi=\varphi * x_{1} T=-a_{(\mathbf{1}, \mathbf{0}, \ldots, 0)} \varphi .
$$

Also folgt aus (10):

$$
x_{1}^{2} T=-a_{(1,0, \ldots, 0)} x_{1} \delta=0 .
$$

$$
\left\|P^{(1,0, \ldots, 0)}(\partial) \varphi\right\|_{2} \leqq(2 / \varepsilon)\left\|e^{\varepsilon\|x\|} P(\partial) \varphi\right\|_{2} .
$$


(12) Es gelte der Satz mit $p=(1,0, \ldots, 0)$ für alle Differentialoperatoren der Ordnung $m$ und es sei $P(\partial)$ ein Differentialoperator der Ordnung $m+1$. Dann ist $P^{(1,0 \ldots, 0)}(\partial)$ ein Differentialoperator der Ordnung $m$, so daß nach Induktionsvoraussetzung gilt:

$$
\left\|P^{(2,0, \ldots, 0)}(\partial) \varphi\right\|_{2} \leqq \frac{2 m}{\varepsilon}\left\|P^{(1,0, \ldots, 0)}(\partial) \varphi\right\|_{2} .
$$

Dazu äquivalent ist

$$
\left\|\varphi * x_{1}^{2} P(\partial) \delta\right\|_{2} \leqq \frac{2 m}{\varepsilon}\left\|\varphi * x_{1} P(\partial) \delta\right\|_{2} .
$$

Aus (10) folgt damit

$$
\left\|\varphi * x_{1} P(\partial) \delta\right\|_{2}^{2} \leqq\left\|e^{\varepsilon\|x\|}(\varphi * P(\partial) \delta)\right\|_{2}\left((2 / \varepsilon)\left\|\varphi * x_{1} P(\partial) \delta\right\|_{2}+(2 m / \varepsilon)\left\|\varphi * x_{1} P(\partial) \delta\right\|_{2}\right)
$$

und

$$
\left\|P^{(1,0, \ldots, 0)}(\partial) \varphi\right\|_{2} \leqq \frac{2(m+1)}{\varepsilon}\left\|e^{\varepsilon\|x\|} P(\partial) \varphi\right\|_{2} .
$$

Bemerkung. Ist ein $a_{i} \neq 0$ für $i \in N_{0}^{n}$ mit $|i|=m$, so gilt

$$
\left|a_{i}\right| i !\|\varphi\|_{2} \leqq\left(\frac{2 m}{\varepsilon}\right)^{m}\left\|e^{\varepsilon\|x\|} P(\partial) \varphi\right\|_{2} .
$$

Also folgt aus $P(\partial) \varphi=0$, da $\|\varphi\|_{2}=0$ und daher $\varphi=0$ gilt, weil $\varphi$ stetig ist. Folglich ist $P(\partial): \mathscr{D} \rightarrow \mathscr{D}$ injektiv, womit ein neuer Beweis von Satz 2 gewonnen ist.

5.4. Wir beweisen

Satz 5. Das durch

$$
\mathscr{D} \rightarrow[0, \infty), \quad \varphi \mapsto\left(\sum_{|j| \leqq r}\left\|e^{\varepsilon\|x\|} \partial^{j} \varphi\right\|_{2}^{2}\right)^{1 / 2}=\left(\sum_{|j| \leqq r} \int e^{2 \varepsilon\|x\|}\left|\partial^{j} \varphi\right|^{2} d x\right)^{1 / 2}
$$

erklärte Funktional ist (für $\varepsilon>0, r \in N_{0}$ ) eine stetige Halbnorm auf $\mathscr{D}$.

Beweis. Es ist zu zeigen, daß für jede kompakte Teilmenge $K$ aus $\boldsymbol{R}^{n}$ die Halbnorm

$$
\mathscr{D}_{K} \rightarrow[0, \infty), \quad \varphi \mapsto\left(\sum_{|j| \leqq r} \int e^{2 \varepsilon\|x\|}\left|\partial^{j} \varphi\right|^{2} d x\right)^{1 / 2}
$$

stetig ist. Es wird für $\varphi \in \mathscr{D}_{K}$

$$
\begin{aligned}
& \sum_{|j| \leqq r} \int e^{2 \varepsilon\|x\|}\left|\partial^{j} \varphi\right|^{2} d x \leqq \sup _{x \in K} e^{2 \varepsilon\|x\|} \sum_{|j| \leqq r} \int\left|\partial^{j} \varphi\right|\left|\partial^{j} \varphi\right| d x \\
& \quad \leqq \sup _{x \in K} e^{2 \varepsilon\|x\|} \sup _{|j| \leqq r} \sup _{x \in K}\left|\partial^{j} \varphi(x)\right| \sum_{|j| \leqq r} \int\left|\partial^{j} \varphi\right| d x \\
& \quad \leqq \sup _{x \in K} e^{2 \varepsilon\|x\|}\left(\sup _{|j| \leqq r} \sup _{x \in K}\left|\partial^{j} \varphi(x)\right|\right)^{2}\left(\sum_{|j| \leqq r} \mu(K)\right),
\end{aligned}
$$

wo $\mu(K)$ das Maß von $K$ ist. 
5.5. Jetzt können wir das Theorem von Malgrange und Ehrenpreis beweisen:

Satz 6. Jeder lineare partielle Differentialoperator mit konstanten Koeffizienten hat eine Fundamentallösung in $\mathscr{D}^{\prime}$.

Beweis. Nach Satz 2 ist die Stetigkeit von $L$ zu zeigen. Dazu wenden wir Satz 4 auf den Differentialoperator $P(-\partial)$ und auf ein $p \in N_{0}^{n}$ mit $|p|=m$ an. Dann ergibt sich

$$
\left|(-1)^{|p|} a_{p}\right| p !\|\varphi\|_{2} \leqq\left(\frac{2 m}{\varepsilon}\right)^{m}\left\|e^{\varepsilon\|x\|} P(-\partial) \varphi\right\|_{2},
$$

also

$$
\left\|\varphi_{2}\right\| \leqq \frac{(2 m / \varepsilon)^{m}}{\max _{|p|=m}\left|a_{p}\right|}\left\|e^{\varepsilon\|x\|} P(-\partial) \varphi\right\|_{2} .
$$

Weiters folgt aus Satz 3 für $a=0$

und daher

$$
|\varphi(0)| \leqq C_{n}\left(\sum_{|j| \leqq[n / 2]+1}\left\|\partial^{j} \varphi\right\|_{2}^{2}\right)^{1 / 2}
$$

sowie

$$
|\varphi(0)| \leqq C_{n}\left(\frac{2 m}{\varepsilon}\right)^{m} \frac{1}{\max _{|p|=m}\left|a_{p}\right|}\left(\sum_{|j| \leqq[n / 2]+1}\left\|e^{\varepsilon\|x\|} \partial^{j}(P(-\partial) \varphi)\right\|_{2}^{2}\right)^{1 / 2},
$$

$$
|L(\psi)| \leqq C\left(\sum_{|j| \leqq[n / 2]+1}\left\|e^{\varepsilon\|x\|} \partial^{j} \psi\right\|_{2}^{2}\right)^{1 / 2}
$$

Wegen Satz 5 ist dann $L$ auf $P(-\partial) \mathscr{D}$ stetig.

Bemerkungen. (1) Das Problem der Existenz einer Fundamentallösung in $\mathscr{D}^{\prime}$ wäre auch gelöst, falls die Existenz einer Fundamentallösung in $\mathscr{S}^{\prime}$ nachgewiesen werden könnte. Dieser Nachweis ist durch Hörmander [5] und Łojasiewicz [7] tatsächlich auch gelungen. Wie jedoch Hörmander [4] bemerkte, haben Fundamentallösungen in $\mathscr{D}^{\prime}$ für gewisse Differentialoperatoren (mit konstanten Koeffizienten) Eigenschaften, die Fundamentallösungen dieser Differentialoperatoren in $\mathscr{S}^{\prime}$ nicht haben können.

(2) Mittels des Satzes von Paley-Wiener-Schwartz ([15], S. 305 ff.) kann man zeigen, daß Fundamentallösungen in $\mathscr{E}^{\prime}$ nicht existieren.

\section{Die Existenz von $L^{2}$-Lösungen linearer partieller Differentialgleichungen mit konstanten Koeffizienten}

Schließlich beweisen wir

Satz 7. Ist $\Omega$ eine beschränkte, nichtleere und offene Teilmenge aus $\boldsymbol{R}^{\boldsymbol{n}}$ und $P(\partial)$ ein Differentialoperator in $\mathscr{D}^{\prime}(\Omega)$ mit konstanten Koeffizienten, so gilt:

$$
P(\partial) L^{2}(\Omega) \supset L^{2}(\Omega) .
$$


Beweis. (Malgrange [10], S. 38.) Es sei für $\varphi \in \mathscr{D}(\Omega)$

Für die durch

$$
P(\partial) \varphi:=\sum_{|j| \leqq m} a_{j} \partial^{j} \varphi .
$$

$$
\hat{\varphi}(x):= \begin{cases}\varphi(x) & x \in \Omega \\ 0 & x \in \complement \Omega\end{cases}
$$

definierte Funktion $\hat{\varphi}$ gilt $\hat{\varphi} \in \mathscr{D}$. Also folgt aus Satz 4 für ein $p \in N_{0}^{n}$ mit $|p|=m$ und für den Differentialoperator

die Ungleichung

$$
P(-\partial) \psi:=\sum_{|j| \leqq m}(-1)^{|j|} a_{j} \partial^{j} \psi \quad \text { für } \quad \psi \in \mathscr{D}
$$

$$
\left|a_{p}\right| p !\|\hat{\varphi}\|_{2} \leqq\left(\frac{2 m}{\varepsilon}\right)^{m}\left\|e^{\varepsilon\|x\|} P(-\partial) \hat{\varphi}\right\|_{2} .
$$

Die Beschränktheit von $\Omega$ liefert

Daher ist

$$
\left|a_{p}\right|\|\hat{\varphi}\|_{2} \leqq\left(\frac{2 m}{\varepsilon}\right)^{m} \sup _{x \in \Omega} e^{\varepsilon\|x\|}\|P(-\partial) \hat{\varphi}\|_{2} .
$$

$$
\|\hat{\varphi}\|_{2} \leqq C\|P(-\partial) \hat{\varphi}\|_{2}
$$

mit einer gewissen Konstante $C$. Weil $\|\hat{\psi}\|_{2}=\|\psi\|_{L^{2}(\Omega)}$ für $\psi \in \mathscr{D}(\Omega)$ und $P(-\partial) \hat{\varphi}=$ $=(P(-\partial) \varphi)^{\wedge}$ ist, gilt

(*)

$$
\|\varphi\|_{L^{2}(\Omega)} \leqq C\|P(-\partial) \varphi\|_{L^{2}(\Omega)} .
$$

Für $f \in L^{2}(\Omega)$ sei $L: P(-\partial) \mathscr{D}(\Omega) \rightarrow C$ durch

$$
L \psi:=(\varphi, \bar{f})_{2} \quad \text { mit } \quad P(-\partial) \varphi=\psi, \quad \varphi \in \mathscr{D}(\Omega)
$$

definiert. Diese Definition ist sinnvoll, da $P(-\partial)$ auf $\mathscr{D}(\Omega)$ wegen $(*)$ injektiv ist. $L$ ist weiters wegen (*) eine stetige Linearform auf dem Untervektorraum $P(-\partial) \mathscr{D}(\Omega)$ von $L^{2}(\Omega)$, wenn dieser mit der von $L^{2}(\Omega)$ induzierten Topologie versehen wird.

Nach dem Satz von Hahn-Banach gibt es dann eine stetige Fortsetzung $\hat{L}$ von $L$. Nach dem Rieszschen Darstellungssatz gibt es weiter ein $v \in L^{2}(\Omega)$ mit

Daher ist für $\varphi \in \mathscr{D}(\Omega)$

$$
\hat{L} g=(g, \bar{v})_{2} \text { für alle } g \in L^{2}(\Omega) .
$$

sowie

$$
\hat{L}(P(-\partial) \varphi)=(P(-\partial) \varphi, \bar{v})_{2},
$$

Somit haben wir

$$
\hat{L}(P(-\partial) \varphi)=L(P(-\partial) \varphi)=(\varphi, \bar{f})_{2} \text {. }
$$

und

$$
(P(-\partial) \varphi, \bar{v})_{2}=(\varphi, \bar{f})_{2}
$$

$$
\langle\varphi, P(\partial) v\rangle=\int \varphi f d x,
$$

wobei $v$ als Distribution aufgefaßt wird. Diese Gleichung aber bedeutet genau in $\mathscr{D}^{\prime}(\Omega)$.

$$
P(\partial) v=f
$$




\section{Literatur}

[1] Dieudonné, J.: Eléments d'analyse. 2. - Gauthier-Villars, Paris, 1969.

[2] Gradshteyn, I. S., und I. W. Ryzhik: Table of integrals, series and products. 4th ed. - Academic Press, New York-London, 1965.

[3] HöRmander, L.: On the theory of general partial differential operators. - Acta Math. 94, $1955,161-248$.

[4] HöRmander, L.: Local and global properties of fundamental solutions. - Math. Scand. 5, 1957, 27-39.

[5] HöRMANDER, L.: On the division of distributions by polynomials. - Ark. Mat. 3, 1958, 555569.

[6] HöRmANDER, L.: Linear partial differential operators. Third revised printing. - Die Grundlehren der mathematischen Wissenschaften 116. Springer-Verlag, Berlin-Heidelberg-New York, 1969.

[7] Lojasiewicz, S.: Sur le problème de la division. - Studia Math. 18, 1959, 87-136.

[8] LounivaAra, I. S., und C. G. Simader: Über nichtelliptische lineare partielle Differentialoperatoren mit konstanten Koeffizienten. - Ann. Acad. Sci. Fenn. Ser. A I 513, 1972, 1-22.

[9] Malgrange, B.: Existence et approximation des solutions des équations aux dérivées partielles et des équations de convolution. - Ann. Inst. Fourier (Grenoble) 6, 1956, 271355.

[10] Malgrange, B.: Introduction aux équations aux dérivées partielles. - Rend. Scuola Internaz. Fis. "E. Fermi", XLV Corso, 32-64.

[11] Rudin, W.: Functional analysis. - McGraw-Hill Book Co., New York, 1973.

[12] Schwartz, L.: Some applications of the theory of distributions. - Lectures on modern mathematics, edited by T. L. Saaty, John Wiley, Inc., New York, 1963, 23-58.

[13] Schwartz, L.: Méthodes mathématiques pour les sciences physiques. - Hermann, Paris, 1965.

[14] Treves, F.: Thèse d'Hörmander I. - Séminaire Bourbaki, exp. 130, 1956, W. A. Benjamin, Inc., New York, 1966.

[15] Treves, F.: Topological vector spaces, distributions and kernels. - Academic Press, New York - London, 1967.

Universität Innsbruck

Institut für Mathematik I

A-6020 Innsbruck

Österreich

Eingegangen am 24. Februar 1978 